\title{
Optical and near-infrared observations of the GRB020405 afterglow ${ }^{\star}$
}

N. Masetti ${ }^{1}$, E. Palazzi ${ }^{1}$, E. Pian ${ }^{1,2}$, A. Simoncelli ${ }^{3}$, L. K. Hunt ${ }^{4}$, E. Maiorano ${ }^{1,3}$, A. Levan ${ }^{5}$, L. Christensen ${ }^{6}$, E. Rol ${ }^{7}$, S. Savaglio ${ }^{8,9}$, R. Falomo ${ }^{10}$, A. J. Castro-Tirado ${ }^{11}$, J. Hjorth ${ }^{12}$, A. Delsanti ${ }^{13}$, M. Pannella ${ }^{14}$, V. Mohan ${ }^{15}$, S. B. Pandey ${ }^{15}$, R. Sagar ${ }^{15}$, L. Amati ${ }^{1}$, I. Burud ${ }^{16}$, J. M. Castro Cerón ${ }^{17}$, F. Frontera ${ }^{1,18}$, A. S. Fruchter ${ }^{16}$, J. P. U. Fynbo ${ }^{19}$, J. Gorosabel ${ }^{11}$, L. Kaper ${ }^{7}$, S. Klose ${ }^{20}$, C. Kouveliotou ${ }^{21}$, L. Nicastro ${ }^{22}$, H. Pedersen ${ }^{12}$, J. Rhoads ${ }^{16}$, I. Salamanca ${ }^{7}$, N. Tanvir ${ }^{23}$, P. M. Vreeswijk ${ }^{7,24}$, R. A. M. J. Wijers ${ }^{7}$, and E. P. J. van den Heuvel ${ }^{7}$

1 Istituto di Astrofisica Spaziale e Fisica Cosmica - Sezione di Bologna, CNR, via Gobetti 101, 40129 Bologna, Italy

2 INAF - Osservatorio Astronomico di Trieste, via G.B. Tiepolo 11, 34131 Trieste, Italy

3 Dipartimento di Astronomia, Università di Bologna, via Ranzani 1, 40126 Bologna, Italy

${ }^{4}$ Istituto di Radioastronomia - Sezione di Firenze, CNR, largo E. Fermi 5, 50125 Florence, Italy

5 Department of Physics and Astronomy, University of Leicester, University Road, Leicester, LE1 7RH, UK

6 Astrophysikalisches Institut, 14482 Potsdam, Germany

7 Institute of Astronomy "Anton Pannekoek”, University of Amsterdam, Kruislaan 403, 1098 SJ Amsterdam, The Netherlands

8 The Johns Hopkins University, 3400 North Charles Street, Bartimore, MD 21218, USA

9 INAF - Osservatorio Astronomico di Roma, via Frascati 33, 00040 Monteporzio Catone, Italy

${ }^{10}$ INAF - Osservatorio Astronomico di Padova, vicolo dell'Osservatorio 5, 35122 Padua, Italy

${ }^{11}$ Instituto de Astrofísica de Andalucía (IAA-CSIC), PO Box 03004, 18080 Granada, Spain

12 Astronomical Observatory, University of Copenhagen, Juliane Maries Vej 30, 2100 Copenhagen Ø, Denmark

13 Observatoire de Paris-Meudon - LESIA, 5 Place Jules Janssen, 92195 Meudon, France

14 Max-Planck-Institut für Extraterrestrische Physik, 85741 Garching, Germany

15 State Observatory, Manora Peak, NainiTal, 263129 Uttaranchal, India

16 Space Telescope Science Institute, 3700 San Martin Drive, Baltimore, MD 21218, USA

17 Real Instituto y Observatorio de la Armada, Sección de Astronomía, 11110 San Fernando-Naval (Cádiz), Spain

18 Dipartimento di Fisica, Università di Ferrara, via Paradiso 12, 44100 Ferrara, Italy

19 Department of Physics and Astronomy, University of Århus, Ny Munkegade, 8000 Århus C, Denmark

20 Thüringer Landessternwarte Tautenburg, 07778 Tautenburg, Germany

21 NASA MSFC, SD-50, Huntsville, AL 35812, USA

22 Istituto di Astrofisica Spaziale e Fisica Cosmica - Sezione di Palermo, CNR, via La Malfa 153, 90146 Palermo, Italy

23 Department of Physical Sciences, University of Hertfordshire, College Lane, Hatfield, Herts AL10 9AB, UK

24 European Southern Observatory, Casilla 19001, Santiago 19, Chile

Received 19 February 2003 / Accepted 27 March 2003

Send offprint requests to: $\mathrm{N}$. Masetti, e-mail: masetti@bo.iasf.cnr.it

* Based on observations made with ESO telescopes at Paranal and La Silla Observatories under programme ID 69.D-0701, with the telescopes TNG, WHT and JKT, operating in the Spanish Observatorio del Roque de los Muchachos of the Instituto de Astrofísica de Canarias, and with the $1 \mathrm{~m}$ telescope of SO in NainiTal, India. 
Abstract. We report on photometric, spectroscopic and polarimetric monitoring of the optical and near-infrared (NIR) afterglow of GRB020405. Ground-based optical observations, performed with 8 different telescopes, started about 1 day after the high-energy prompt event and spanned a period of $\sim 10$ days; the addition of archival HST data extended the coverage up to $\sim 150$ days after the GRB. We report the first detection of the afterglow in NIR bands. The detection of Balmer and oxygen emission lines in the optical spectrum of the host galaxy indicates that the GRB is located at redshift $z=0.691$. Fe II and Mg II absorption systems are detected at $z=0.691$ and at $z=0.472$ in the afterglow optical spectrum. The latter system is likely caused by absorbing clouds in the galaxy complex located $\sim 2$ " southwest of the GRB020405 host. Hence, for the first time, the galaxy responsible for an intervening absorption line system in the spectrum of a GRB afterglow is spectroscopically identified. Optical and NIR photometry of the afterglow indicates that, between 1 and 10 days after the GRB, the decay in all bands is consistent with a single power law of index $\alpha=1.54 \pm 0.06$. The late-epoch VLT $J$-band and HST optical points lie above the extrapolation of this power law, so that a plateau (or "bump") is apparent in the VRIJ light curves at 10-20 days after the GRB. The light curves at epochs later than day $\sim 20$ after the GRB are consistent with a power-law decay with index $\alpha^{\prime}=1.85 \pm 0.15$. While other authors have proposed to reproduce the bump with the template of the supernova (SN) 1998bw, considered the prototypical "hypernova", we suggest that it can also be modeled with a SN having the same temporal profile as the other proposed hypernova SN2002ap, but 1.3 mag brighter at peak, and located at the GRB redshift. Alternatively, a shock re-energization may be responsible for the rebrightening. A single polarimetric $R$-band measurement shows that the afterglow is polarized, with $P=1.5 \pm 0.4 \%$ and polarization angle $\theta=172^{\circ} \pm 8^{\circ}$. Broad-band optical-NIR spectral flux distributions show, in the first days after the GRB, a change of slope across the $J$ band which we interpret as due to the presence of the electron cooling frequency $v_{\mathrm{c}}$. The analysis of the multiwavelength spectrum within the standard fireball model suggests that a population of relativistic electrons with index $p \sim 2.7$ produces the optical-NIR emission via synchrotron radiation in an adiabatically expanding blastwave, with negligible host galaxy extinction, and the X-rays via Inverse Compton scattering off lower-frequency afterglow photons.

Key words. gamma rays: bursts - radiation mechanisms: non-thermal - line: identification - cosmology: observations

\section{Introduction}

GRB020405 was detected by the Third InterPlanetary Network (IPN) on 2002 April 5.02877 UT with a duration of $\sim 40 \mathrm{~s}$ and localized to an error box of 75 square arcmin size. In the $25-100 \mathrm{keV}$ band it had a total fluence of $\sim 3 \times$ $10^{-5} \mathrm{erg} \mathrm{cm}^{-2}$ and a peak flux of $\sim 10^{-6} \mathrm{erg} \mathrm{cm}^{-2} \mathrm{~s}^{-1}$ (Hurley et al. 2002). This GRB was also observed by the GRBM onboard BeppoSAX, with a duration of $\sim 60 \mathrm{~s}$ in the $40-700 \mathrm{keV}$ band and a $50-700 \mathrm{keV}$ fluence of $\sim 4 \times 10^{-5} \mathrm{erg} \mathrm{cm}^{-2}$ (Price et al. 2003).

Approximately $18 \mathrm{hrs}$ after the GRB, Price et al. (2002a, 2003) detected a relatively bright $(R \sim 18.5)$ source within the IPN error box which was not present on the DSS-II red plate. This Optical Transient (OT), located at coordinates (J2000) $\mathrm{RA}=13^{\mathrm{h}} 58^{\mathrm{m}} 03.12$; Dec $=-31^{\circ} 22^{\prime} 22^{\prime \prime} .2$ (with an error of 0.3 along both directions), was confirmed by subsequent observations and identified as the afterglow of GRB020405 (Castro-Tirado et al. 2002; Palazzi et al. 2002; Hjorth et al. 2002; Price et al. 2002b; Gal-Yam et al. 2002a; Covino et al. 2002a,b). Optical spectroscopy allowed Masetti et al. (2002a) and Price et al. (2003) to determine the redshift of the GRB, $z=0.691$ (see also Sect. 3.3).

A counterpart to the GRB has been detected also at radio and X-ray wavelengths (Mirabal et al. 2003; Berger et al. 2003). By fitting to the optical light curves a power law $F(t) \propto$ $t^{-\alpha}$, typical of GRB afterglows, Price et al. (2002b) and Covino et al. (2002b) found $\alpha \sim 1.26$ and $\alpha=1.52 \pm 0.12$, respectively, from observations performed within 3 days after the GRB. By using a data set spanning $~ 5$ days, Price et al. (2003) found instead that a better fit to the early optical afterglow light curve was obtained with a smoothly broken power law with decay indices $\alpha_{1} \sim 0.9$ and $\alpha_{2} \sim 1.9$ before and after a break which occurred $\sim 1.7$ days after the GRB, respectively. On the other hand, Bersier et al. (2003) found no evidence of a break between 1.24 and 4.3 days after the GRB in their $R$-band data, which followed a single power-law decay with index $\alpha \sim 1.7$.
Polarimetric measurements by Covino et al. (2003) and Bersier et al. (2003) suggest early rapid variability of the percentage of linear polarization between values of $\sim 1.5 \%$ and $\sim 10 \%$.

Price et al. (2003), using late-time HST observations covering the time interval between 20 and 140 days after the highenergy prompt event, found a "red bump", or flattening, in the optical light curves of the OT and suggested that this could be due to an emerging supernova (SN) component, which they modeled with the template of SN1998bw located at the redshift of GRB020405 and further dimmed by about $0.5 \mathrm{mag}$. A similar assumption was made by Dado et al. (2002) in their modeling of the GRB020405 optical afterglow within the cannonball picture.

Optical observations also revealed the presence of a relatively large (about $2^{\prime \prime} \times 1^{\prime \prime}$, elongated in the N-S direction) nebulosity located $\sim 2^{\prime \prime}$ southwest of the OT and interpreted as its putative host galaxy (Hjorth et al. 2002).

In this paper we report on optical imaging, spectroscopy and polarimetry, along with the first detection in the nearinfrared (NIR) bands of the GRB020405 afterglow. The data were acquired, in the framework of the GRACE ${ }^{1}$ collaboration, at the ESO telescopes of Cerro Paranal and La Silla (Chile) starting on 6 April 2002, i.e., $\sim 1$ day after the high-energy prompt event. Observations from the 1-m SO telescope, WHT and TNG are also included, along with the reanalysis of the public HST observations of this GRB.

The paper is organized as follows: Sect. 2 describes the optical and NIR observations and the data analysis; the results are reported in Sect. 3 and discussed in Sect. 4; in Sect. 5 we report our conclusions. Throughout the paper we assume a cosmology with $H_{0}=65 \mathrm{~km} \mathrm{~s}^{-1} \mathrm{Mpc}^{-1}, \Omega_{\Lambda}=0.7$ and $\Omega_{\mathrm{m}}=0.3$; also, when not otherwise stated, uncertainties will be reported at $1 \sigma$ confidence level, and upper limits at $3 \sigma$ confidence level.

1 GRB Afterglow Collaboration at ESO: see the web page http://zon.wins. uva.nl/grb/ grace 


\section{Observations and data reduction}

\subsection{Optical and NIR photometry}

Optical BVRI observations in the Johnson-Cousins photometric system were performed with VLT-Melipal plus FORS1, VLT-Yepun plus FORS2, NTT plus SUSI2 and EMMI, and 1.54-metre Danish telescope plus DFOSC.

FORS1 is equipped with a $2048 \times 2048$ pixels Tektronix CCD which covers a 6.8 $\times 6.8$ field in the standard resolution imaging mode with a scale of 0.2 pix $^{-1}$; FORS2 is equipped with a mosaic of two $2048 \times 4096$ pixels MIT CCDs, usually operating in $2 \times 2$ standard binning mode, thus covering a field with size $6.8 \times 6 ! 8$ with a spatial resolution of $0.25 \mathrm{pix}^{-1}$ in the standard resolution imaging mode. SUSI 2 incorporates a mosaic of two $2048 \times 4096$ pixels EEV CCDs which cover a field of $5.5 \times 5.5$, corresponding to 0. '08 pix $^{-1}$; EMMI (Red Arm) carried until 14 May 2002 a $2048 \times 2047$ pixels Tektronix CCD with a scale of 0.27 pix $^{-1}$ and a field coverage of $9.15 \times 8.6$ in size. DFOSC is equipped with a $2048 \times 2048$ pixels CCD covering a 13 '. $\times 13$ ! 7 field, thus securing a spatial resolution of $0.39 \mathrm{pix}^{-1}$.

Optical observations were also obtained on 5 and 6 April 2002 ( $I$ band) at the $1.0 \mathrm{~m}$ Sampurnanand telescope of SO located in Nainital (India), and on 6 April 2002 at the Canary Islands (Spain) with the $4.2 \mathrm{~m}$ WHT plus PFIP ( $U B V R I$ bands) and with the $3.58 \mathrm{~m}$ TNG plus DOLoReS ( $V$ band). The $1.0 \mathrm{~m}$ SO telescope carries a $2048 \times 2048$ pixels CCD, with a $13^{\prime} \times 13^{\prime}$ field of view; in its standard $2 \times 2$ binning mode it has a spatial resolution of $0.76 \mathrm{pix}^{-1}$. The imaging camera PFIP carries two $2100 \times 4200 \mathrm{EEV}$ CCDs which cover a field of view of $16 ! 2 \times 16 ! 2$, giving a plate scale of $0.24 \mathrm{pix}^{-1}$; the spectrophotometer DOLoReS carries a $2048 \times 2048$ pixels Loral CCD which can image a field of 9.5 $\times 9.5$ with a scale of $0.275 \mathrm{pix}^{-1}$.

NIR imaging was obtained at ESO NTT with SofI $(J, H$ and $K_{\mathrm{s}}$ bands) and at VLT-Antu with ISAAC ( $J_{\mathrm{s}}$ band). The SofI infrared spectrograph and imaging camera works in the $0.9-2.5 \mu \mathrm{m}$ NIR range by using a Hawaii $1024 \times 1024$ pixel $\mathrm{HgCdTe}$ array. In the small-field imaging mode the plate scale is $0{ }^{\prime} 144 \mathrm{pix}^{-1}$ and the corresponding field of view is $2.4 \times 2$ I $^{\prime} 4$. ISAAC is equipped, in the $0.9-2.5 \mu \mathrm{m}$ range, with a Rockwell Hawaii $1024 \times 1024$ pixel $\mathrm{HgCdTe}$ array which has a scale of $0 .^{\prime} 148$ pix $^{-1}$ and secures imaging with a field of view of $2 ! 5 \times 2.5$. The $J_{\mathrm{s}}$ filter is centered at $1.24 \mu \mathrm{m}$ and has a full width at half maximum of $0.16 \mu \mathrm{m}$; its overall response is $25 \%$ lower than the $J$ filter. This is confirmed by comparing the nightly zero-point coefficients of $J$ and $J_{\mathrm{s}}$ frames in our data set. We thus reported the ISAAC $J_{\mathrm{s}}$ magnitudes to the standard $J$ band by increasing the observed $J_{\mathrm{s}}$-band flux densities by $25 \%$. In order to allow for sky subtraction, the total integration time of each NIR pointing was split into dithered images of $15 \mathrm{~s}$ each when using SofI, and of $30 \mathrm{~s}$ each during ISAAC observations. In both cases the dithering was $40^{\prime \prime}$ between consecutive images.

The complete log of our optical and NIR imaging observations is reported in Table 1.

Optical images were bias-subtracted and flat-fielded with the standard cleaning procedure. In some cases (especially at late epochs), frames taken on the same night in the same band were summed together in order to increase the signal-to-noise ratio. In Fig. 1 we report the $R$-band image of the field of the GRB020405 counterpart obtained on 6 April 2002 with the 1.54-m Danish telescope plus DFOSC.

Since the close environment of the afterglow is quite crowded (see Figs. 3 and 6), we chose standard Point Spread Function (PSF) fitting, rather than simple aperture, photometry. We used the DAOPHOT II image data analysis package PSF-fitting algorithm (Stetson 1987) running within MIDAS ${ }^{2}$. A two-dimensional Gaussian profile with two free parameters (the half width at half maxima along $x$ and $y$ coordinates of each frame) was modeled on at least 5 unsaturated bright stars in each image. The errors associated with the measurements reported in Table 1 represent statistical uncertainties obtained with the standard PSF-fitting procedure.

The BVRI zero-point calibration was performed by using several standard fields (Landolt 1992) taken under photometric conditions at several ESO telescopes. The single Gunn $i$ image (6 April 2002) was calibrated using the $I$-band secondary standards, given that the widths, the reference wavelengths and the flux density normalizations of the two filters are very similar (Fukugita et al. 1995). However, in order to account for small differences between the filters, we added in quadrature a $3 \%$ uncertainty to the $I$-band magnitude error obtained from the Gunn $i$ observation.

Concerning the single $U$-band measurement taken at WHT, no standard field was acquired on the same night due to nonphotometric conditions of the sky. The calibration in this band was then performed on 10 May 2002 at the 1.0-m JKT, located in the Canary Islands and equipped with a SITe2 CCD which has $2048 \times 2048$ pixels and an image scale of 0.33 pix $^{-1}$. Observations of the GRB field and of some Landolt (1992) fields were acquired in $U$ and $B$ to determine the CCD color term.

We then selected 6 stars of different brightness in the GRB020405 field and we used them to determine the OT optical BVRI magnitudes (see also Simoncelli et al. 2002). Two additional stars were used for the calibration in the $U$ band. All these stars are indicated in Fig. 1 and their magnitudes are listed in Table 2. We find this magnitude calibration to be accurate to within $3 \%$. We note that the $B$ and $R$ magnitudes of field stars as reported in the USNO-A2.0 catalog ${ }^{3}$ differ by $\sim 0.6$ mag with respect to our calibration, which is however consistent with that of Bersier et al. (2003) and Price et al. (2003).

Public HST/WFPC2 observations of GRB020405 taken in three filters (F555W, F702W and F814W) have been included in our analysis (see also Price et al. 2003). All HST data were retrieved from the multimission archive ${ }^{4}$ after "On-The-Fly" calibration had been performed. Dithered images were then combined and cosmic-ray cleaned using the drizzle method (Fruchter \& Hook 2002). The images were drizzled onto a final

\footnotetext{
${ }^{2}$ MIDAS (Munich Image Data Analysis System) is developed, distributed and maintained by ESO (European Southern Observatory) and is available at http://www.eso.org/projects/esomidas/

3 available at http://www. nofs.navy.mil/

${ }^{4}$ see http://archive.stsci.edu/
} 
Table 1. Journal of the GRB020405 afterglow ground-based optical and NIR photometric observations. Magnitudes are not corrected for Galactic interstellar absorption.

\begin{tabular}{|c|c|c|c|c|c|c|}
\hline $\begin{array}{l}\text { Mid-exposure } \\
\text { time (UT) }\end{array}$ & Telescope & Instrument & Filter & $\begin{array}{l}\text { Total exposure } \\
\text { time (s) }\end{array}$ & $\begin{array}{l}\text { Seeing } \\
(\operatorname{arcsec})\end{array}$ & Magnitude \\
\hline 2002 Apr. 5.812 & $1 \mathrm{~m} \mathrm{SO}$ & $\mathrm{CCD}$ & $I$ & $2 \times 600$ & 2.6 & $19.30 \pm 0.06$ \\
\hline 2002 Apr. 6.069 & TNG & DOLoRes & $V$ & $2 \times 300$ & 1.8 & $20.66 \pm 0.02^{*}$ \\
\hline 2002 Apr. 6.013 & WHT & PFIP & $R$ & 300 & 2.3 & $20.17 \pm 0.03$ \\
\hline 2002 Apr. 6.109 & WHT & PFIP & $U$ & 900 & 2.2 & $20.48 \pm 0.06$ \\
\hline 2002 Apr. 6.118 & WHT & PFIP & $B$ & 600 & 2.5 & $21.21 \pm 0.03$ \\
\hline 2002 Apr. 6.124 & WHT & PFIP & $V$ & 300 & 2.0 & $20.73 \pm 0.03$ \\
\hline 2002 Apr. 6.129 & WHT & PFIP & $R$ & 300 & 2.8 & $20.28 \pm 0.04$ \\
\hline 2002 Apr. 6.134 & WHT & PFIP & $I$ & 300 & 3.2 & $19.61 \pm 0.08$ \\
\hline 2002 Apr. 6.136 & $1.54 \mathrm{D}$ & DFOSC & $R$ & 600 & 1.4 & $20.38 \pm 0.02$ \\
\hline 2002 Apr. 6.186 & Melipal & FORS1 & $R$ & 60 & 0.7 & $20.48 \pm 0.01$ \\
\hline 2002 Apr. 6.197 & Melipal & FORS1 & $R$ & 30 & 0.8 & $20.46 \pm 0.01$ \\
\hline 2002 Apr. 6.211 & $1.54 \mathrm{D}$ & DFOSC & $R$ & 600 & 1.4 & $20.44 \pm 0.02$ \\
\hline 2002 Apr. 6.220 & $1.54 \mathrm{D}$ & DFOSC & $V$ & 600 & 1.4 & $20.81 \pm 0.02$ \\
\hline 2002 Apr. 6.220 & NTT & SofI & $H$ & 300 & 1.0 & $18.07 \pm 0.07$ \\
\hline 2002 Apr. 6.224 & NTT & SofI & $J$ & 300 & 1.0 & $18.69 \pm 0.06$ \\
\hline 2002 Apr. 6.230 & $1.54 \mathrm{D}$ & DFOSC & $B$ & 600 & 1.4 & $21.31 \pm 0.03$ \\
\hline 2002 Apr. 6.230 & NTT & SofI & $K_{\mathrm{s}}$ & 600 & 0.9 & $17.34 \pm 0.06$ \\
\hline 2002 Apr. 6.234 & Melipal & FORS1 & $R$ & 20 & 0.8 & $20.53 \pm 0.02$ \\
\hline 2002 Apr. 6.246 & $1.54 \mathrm{D}$ & DFOSC & Gunn $i$ & 600 & 1.1 & $19.88 \pm 0.02$ \\
\hline 2002 Apr. 6.797 & $1 \mathrm{~m} \mathrm{SO}$ & $\mathrm{CCD}$ & $I$ & $5 \times 600$ & 2.5 & $>20.0$ \\
\hline 2002 Apr. 7.132 & $1.54 \mathrm{D}$ & DFOSC & $R$ & 600 & 1.2 & $21.20 \pm 0.04$ \\
\hline 2002 Apr. 7.240 & $1.54 \mathrm{D}$ & DFOSC & $R$ & 600 & 0.9 & $21.30 \pm 0.06$ \\
\hline 2002 Apr. 7.249 & $1.54 \mathrm{D}$ & DFOSC & $V$ & 600 & 1.1 & $21.79 \pm 0.05$ \\
\hline 2002 Apr. 7.266 & $1.54 \mathrm{D}$ & DFOSC & $B$ & 600 & 1.2 & $22.29 \pm 0.07$ \\
\hline 2002 Apr. 7.270 & NTT & SofI & $H$ & 600 & 0.8 & $18.90 \pm 0.07$ \\
\hline 2002 Apr. 7.278 & NTT & SofI & $J$ & 600 & 0.8 & $19.55 \pm 0.06$ \\
\hline 2002 Apr. 7.281 & Melipal & FORS1 & $V$ & $2 \times 30$ & 0.7 & $21.95 \pm 0.04$ \\
\hline 2002 Apr. 7.294 & NTT & SofI & $K_{\mathrm{s}}$ & 1800 & 0.7 & $18.18 \pm 0.06$ \\
\hline 2002 Apr. 7.381 & $1.54 \mathrm{D}$ & DFOSC & $R$ & 600 & 1.2 & $21.32 \pm 0.06$ \\
\hline 2002 Apr. 8.280 & NTT & SUSI2 & $V$ & $10 \times 60$ & 0.8 & $22.49 \pm 0.02$ \\
\hline 2002 Apr. 8.294 & NTT & SUSI2 & $R$ & $10 \times 60$ & 0.8 & $22.09 \pm 0.03$ \\
\hline 2002 Apr. 8.310 & NTT & SUSI2 & $I$ & $5 \times 60+3 \times 300$ & 0.8 & $21.44 \pm 0.02$ \\
\hline 2002 Apr. 9.205 & NTT & EMMI & $R$ & $2 \times 300$ & 1.0 & $22.23 \pm 0.04$ \\
\hline 2002 Apr. 9.194 & NTT & EMMI & $V$ & $2 \times 300$ & 1.0 & $22.76 \pm 0.06$ \\
\hline 2002 Apr. 9.215 & NTT & EMMI & $I$ & $2 \times 300$ & 1.0 & $21.70 \pm 0.06$ \\
\hline 2002 Apr. 12.097 & Yepun & FORS2 & $V$ & $3 \times 180$ & 0.7 & $23.59 \pm 0.04$ \\
\hline 2002 Apr. 12.107 & Yepun & FORS2 & $R$ & $3 \times 180$ & 0.7 & $23.09 \pm 0.04$ \\
\hline 2002 Apr. 15.238 & Melipal & FORS1 & $V$ & $2 \times 180$ & 0.7 & $23.99 \pm 0.07$ \\
\hline 2002 Apr. 15.244 & Melipal & FORS1 & $R$ & $2 \times 180$ & 0.6 & $23.36 \pm 0.04$ \\
\hline 2002 Apr. 15.250 & Melipal & FORS1 & $I$ & $2 \times 180$ & 0.6 & $22.53 \pm 0.06$ \\
\hline 2002 Apr. 27.193 & Antu & ISAAC & $J_{\mathrm{s}}$ & 3600 & 0.5 & $22.4 \pm 0.1$ \\
\hline 2002 May 8.193 & Antu & ISAAC & $J_{\mathrm{s}}$ & 3600 & 0.6 & $>22.7$ \\
\hline
\end{tabular}

${ }^{*}$ A preliminary analysis of this observation was presented in Palazzi et al. (2002). 
Table 2. Optical magnitudes of selected GRB020405 field stars as indicated in Fig. 1. Values are not reported for cases in which the stars are either saturated or too faint for reliable calibration.

\begin{tabular}{cccccc}
\hline \hline Star & $U$ & $B$ & $V$ & $R$ & $I$ \\
\hline 1 & - & $19.57 \pm 0.01$ & $18.69 \pm 0.01$ & $18.17 \pm 0.01$ & $17.55 \pm 0.01$ \\
2 & - & $19.84 \pm 0.01$ & $19.01 \pm 0.01$ & $18.48 \pm 0.01$ & $17.87 \pm 0.01$ \\
3 & - & $19.34 \pm 0.01$ & $18.85 \pm 0.01$ & $18.50 \pm 0.02$ & $18.12 \pm 0.01$ \\
4 & - & $20.09 \pm 0.02$ & $19.49 \pm 0.01$ & $19.14 \pm 0.02$ & $18.72 \pm 0.01$ \\
5 & - & $18.18 \pm 0.01$ & $17.92 \pm 0.01$ & $17.72 \pm 0.01$ & $17.45 \pm 0.01$ \\
6 & - & $20.45 \pm 0.02$ & $19.35 \pm 0.01$ & $18.67 \pm 0.01$ & $18.00 \pm 0.01$ \\
A & $15.76 \pm 0.01$ & $16.00 \pm 0.02$ & - & - & - \\
B & $18.35 \pm 0.03$ & $17.74 \pm 0.02$ & - & - & - \\
\hline
\end{tabular}

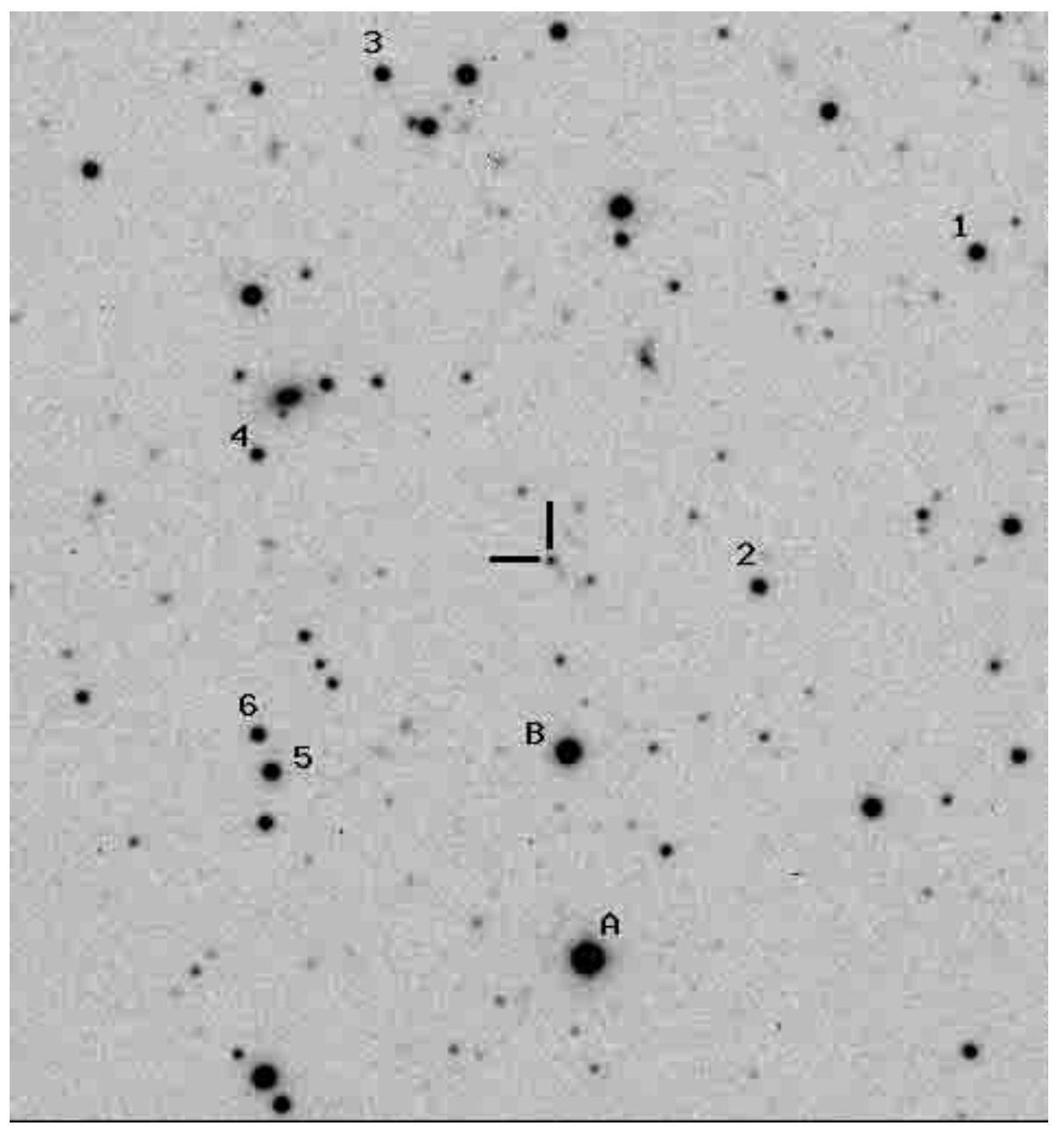

Fig. 1. $R$-band image of the field of GRB020405 acquired with the 1.54-metre Danish telescope plus DFOSC on 2002 April 6.136 UT at ESO-La Silla. The OT is at the centre of the image, indicated with the tick marks. North is at top, East is to the left; the field size is about $3^{\prime} \times 3^{\prime}$. Numbers and letters indicate the reference stars with magnitudes reported in Table 2. output grid with pixels half the size of the native WPFC2 pixels using a pixfrac parameter of 0.7 .

The images acquired with filters F555W and F702W on August 23, 2002, i.e., 140 days after the GRB, do not show substantial pointlike emission at the OT position; therefore they were used to evaluate the host galaxy contribution underlying the afterglow. This could not be done for the F814W data as no images were acquired with this filter during the last HST visit. Therefore, in order to estimate the magnitude of the host underlying the OT position in the F814W frames, we created a PSF using the Tiny Tim software (Krist 1993). This PSF was subsampled and convolved to mimic the effect of the drizzle.
The PSF was then subtracted from the position of the OT in the June 2002 F814W HST images to the level where the remaining surface brightness was entirely due to the host. These PSF-subtracted images were then used as a template to determine and subtract the host galaxy contribution in all previous (space- and ground-based) observations made in that band. The accuracy of this method was checked by applying it to the June 2002 images acquired with filters F555W and F702W, and by comparing the results with those obtained using the August 2002 data. We found that the host magnitudes measured at the OT position with the two methods (i.e., direct host contribution measurement on one hand, and PSF modeling plus 
Table 3. HST-WFPC2 optical magnitudes of the GRB020405 OT. The values are corrected for the host galaxy contribution, but not for the Galactic absorption.

\begin{tabular}{lcc}
\hline \hline Mid-exposure date (UT) & Filter & Magnitude \\
\hline 2002 Apr. 24.272 & F555W & $25.05 \pm 0.04$ \\
2002 May 5.631 & F555W & $25.83 \pm 0.05$ \\
2002 Jun. 2.711 & F555W & $27.25 \pm 0.15$ \\
2002 Aug. 23.246 & F555W & $>27.5$ \\
& & \\
2002 Apr. 28.452 & F702W & $23.99 \pm 0.02$ \\
2002 May 1.620 & F702W & $24.31 \pm 0.02$ \\
2002 May 3.625 & F702W & $24.38 \pm 0.02$ \\
2002 Jun. 1.614 & F702W & $25.91 \pm 0.12$ \\
2002 Aug. 23.451 & F702W & $>26.7$ \\
2002 Apr. 26.275 & F814W & $23.13 \pm 0.01$ \\
2002 May 1.486 & F814W & $23.45 \pm 0.02$ \\
2002 Jun. 9.560 & F814W & $25.42 \pm 0.15$ \\
\hline
\end{tabular}

subtraction on the other) coincide within the uncertainties. This made us confident that the host contribution, as derived with the PSF-subtraction method, was correct and could be applied to the F814W images.

OT magnitudes were then measured on each available HST image with standard aperture photometry, and were calibrated to the Vega system using the published zeropoints for the WFPC2 (Biretta et al. 2002). We assumed that the conversion from the WFPC2 to the Johnson-Cousins system did not induce significant errors on the photometry, as also stated in Fukugita et al. (1995). Indeed, the resulting OT magnitudes, reported in Table 3, are consistent with the flux values independently reported by Price et al. (2003). The only discrepancy is found in the F814W filter data and arises from the fact that those authors assumed no OT contribution in the June 2002 HST image acquired with that filter, so that was used directly as the template for the host galaxy subtraction. In fact, if we subtract, for that HST filter, the June 2002 magnitude of the OT plus underlying host from those obtained in the previous visits, we obtain flux density values consistent with those reported in Table 3 of Price et al. (2003).

The reduction of the NIR images was performed with IRAF and the STSDAS packages ${ }^{5}$. Each image was reduced by first subtracting a mean sky, obtained from the median of a number of images acquired just before and after each processed frame. Before the frames were used for sky subtraction, stars in them were eliminated by a background interpolation algorithm (imedit) combined with an automatic "star finder" (daofind). Then, a differential dome flatfield correction was applied; next, the telescope dithering was measured from the offsets of field

\footnotetext{
${ }^{5}$ IRAF is the Image Analysis and Reduction Facility made available to the astronomical community by the National Optical Astronomy Observatories, which are operated by AURA, Inc., under contract with the U.S. National Science Foundation. STSDAS is distributed by the Space Telescope Science Institute, which is operated by the Association of Universities for Research in Astronomy (AURA), Inc., under NASA contract NAS 5-26555.
}

objects in each image and the images were averaged together using inter-pixel shifts. Also for the NIR images we used PSFfitting photometry following the same procedure adopted in the optical. We calibrated the NIR photometry using stars selected from the NICMOS Standards List (Persson et al. 1998) and determined the NIR zero-point coefficients for each observation. The standard stars were observed in five positions on the detector, and their images were reduced in the same way as those of the GRB field. Formal photometric accuracy based only on the standard stars observations is typically better than $3 \%$. Our calibration is fully consistent with the results of the 2MASS survey $^{6}$ (Skrutskie et al. 1997) for the GRB020405 field.

Next we evaluated the Galactic absorption in the optical and NIR bands along the direction of GRB020405 using the galactic dust infrared maps by Schlegel et al. (1998); from these data we obtained a color excess $E(B-V)=0.055$. By applying the relation by Cardelli et al. (1989), we derived $A_{U}=0.27$, $A_{B}=0.22, A_{V}=0.17, A_{R}=0.14, A_{I}=0.10, A_{J}=0.05$, $A_{H}=0.03$ and $A_{K_{\mathrm{s}}}=0.02$.

\subsection{Optical spectroscopy}

Two series of spectra with total exposure times of $30 \mathrm{~min}$ and 60 min were obtained on 2002 April 6.213 and 7.310 UT, respectively, with VLT-Melipal+FORS1. The first series was acquired using Grism 150I plus order separator OG590, which avoids overlapping of spectral orders over a given wavelength; this limited the spectral range to $6000-9000 \AA$. For the second series the Grism $300 \mathrm{~V}$ with no order separator was used: this allowed a wider spectral coverage (3500-9000 A) and a spectral resolution higher by about a factor of two. The slit width was $1^{\prime \prime}$ for both observations and these setups secured final dispersions of $5.5 \AA \mathrm{pix}^{-1}$ for the first series and of $2.6 \AA \mathrm{pix}^{-1}$ for the second. In the second spectroscopic pointing the slit was rotated by about $40^{\circ}$ with respect to the N-S direction in order to include both the OT and the galaxy located $\sim 2$ " southwest of it (Hjorth et al. 2002).

The spectra, after correction for flat-field and bias, were background subtracted and optimally extracted (Horne 1986) using IRAF. He-Ne-Ar and $\mathrm{Hg}-\mathrm{Cd}$ lamps were used for wavelength calibration; both spectroscopic runs were then fluxcalibrated by using the spectroscopic standards LTT 6248 and Hiltner 600 (Hamuy et al. 1992, 1994) for the April 6 and 7 observations, respectively. Finally, spectra taken within the same night were stacked together to increase the $\mathrm{S} / \mathrm{N}$ ratio. The correctness of the wavelength and flux calibrations was checked against the position of night sky lines and the photometric data collected around the epoch at which the spectra were acquired, respectively. The typical errors were $0.3 \AA$ for the wavelength calibration and $10 \%$ for the flux calibration.

\subsection{Optical polarimetry}

Linear polarimetry in the $R$ band was accomplished on 2002 April 6.236 UT at VLT-Melipal+FORS1 under an average seeing of $0 .{ }^{\prime} 8$. Data were obtained by using a Wollaston prism and

\footnotetext{
${ }^{6}$ available at http://www.ipac. caltech.edu/2mass/
} 


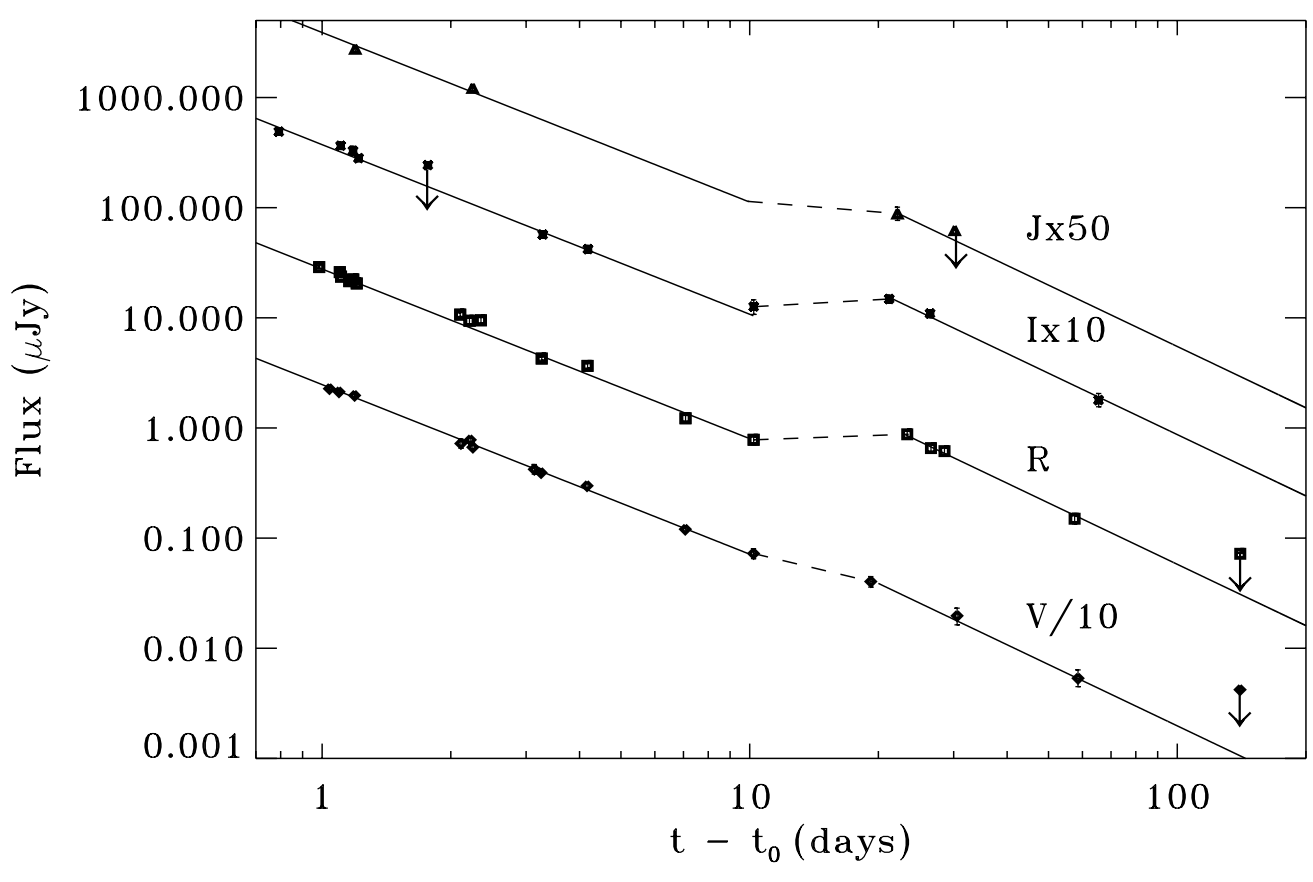

Fig. 2. VRIJ light curves of the GRB020405 afterglow. Different symbol styles indicate different bands. Data are corrected for the underlying host galaxy contribution and for Galactic absorption. The light curves were rescaled in flux for clarity using the factors indicated in the figure. A flattening between day 10 and 20 after the GRB, more evident in the RIJ bands, is detected. The light curves are best fitted using a power law with $\alpha=$ 1.54 (up to 10 days after the GRB) and a power law with $\alpha^{\prime}=1.85$ (after day 20). a half-wavelength, phase-retarder plate. The Wollaston prism separates the incident light into an ordinary and an extraordinary component, while the phase-retarder plate determines which of the Stokes parameters is measured ( $U$ or $Q$ ). For each image, a mask producing $22^{\prime \prime}$-wide parallel strips was used to avoid overlap of the ordinary and extraordinary components. The observation therefore consisted of four exposures centered at the position of the OT, with the phase-retarder plate at $0^{\circ}$, $22.5,45^{\circ}$ and $67^{\circ} .5$. Each angle was imaged with an exposure time of $300 \mathrm{~s}$, for a total exposure time of $1200 \mathrm{~s}$ over the four angles. Image reduction and analysis was performed as described in Sect. 2.1 for the optical photometry. No significant variation among the values of the $Q$ and $U$ parameters of individual field stars was noted, therefore we computed the average values of these quantities and subtracted them from the corresponding parameters of the OT to remove the instrumental and (local) interstellar polarization. We also checked that the polarization of the selected field stars did not systematically vary with their position on the CCD or with their magnitude. The polarization angle $\theta$ was calibrated by using the polarized standard Vela1_95 (Szeifert 2001). The unpolarized standard WD 1620-315 (Szeifert 2001) was used to check the instrumental polarization, which was found to be negligible.

\section{Results}

The optical (see Fig. 1) and NIR images show a point-like source at the OT position indicated by Price et al. (2002a, 2003). The transient behaviour observed in both spectral ranges (see Table 1) confirms the afterglow nature of the source.

\subsection{Light curves}

In the analysis of the optical and NIR light curves of the GRB020405 we added to our data set the points published by Covino et al. (2003), whose photometry can be connected with ours. We first considered the ground-based data. These may be contaminated by the underlying host galaxy complex. Thus, in order to correct for its contribution to the VRI magnitudes of the afterglow, we followed two approaches: (i) we fit the light curves with a single power law plus constant ( $F \propto t^{-\alpha}+$ const); (ii) we measured, on the latest available (OTsubtracted) HST images, the contribution of the host galaxy complex within an aperture radius matching the ground-based telescopes PSFs and subtracted it from our photometric measurements. The two methods gave results which are consistent within the errors, and showed that the host contribution to the OT luminosity was of the order of few percent (and thus negligible if compared with the uncertainties) in the first days of observation.

Concerning the $J$-band data, in the VLT-ISAAC image of 8 May 2002 we did not detect a point-like source at the position of the afterglow, despite the depth and good resolution of the image. Therefore we evaluated the host galaxy contribution in the $J$-band by using aperture photometry centered at the OT position and with radius equal to the FWHM of the image PSF. This can be conservatively considered as an upper limit to the OT flux.

No late-time HST or ground-based data are available for other optical or NIR filters. Therefore, no host-subtraction could be done for bands different than VRIJ. Since the host contribution could be evaluated only for these four bands, only the corresponding light curves are reported in Fig. 2.

The host-subtracted VRI light curves between 1 and 10 days after the GRB decay according to single power laws whose temporal indices are not significantly different from filter to filter. Their average value is $\alpha=1.54 \pm 0.06$. The $B$ light curve, which consists of few, early epoch points, is fitted by the same temporal power law. By modeling the light curves using a smoothly broken power law as described by Beuermann et al. (1999) we obtain a worse fit (i.e., with a larger reduced $\chi^{2}$ ), 


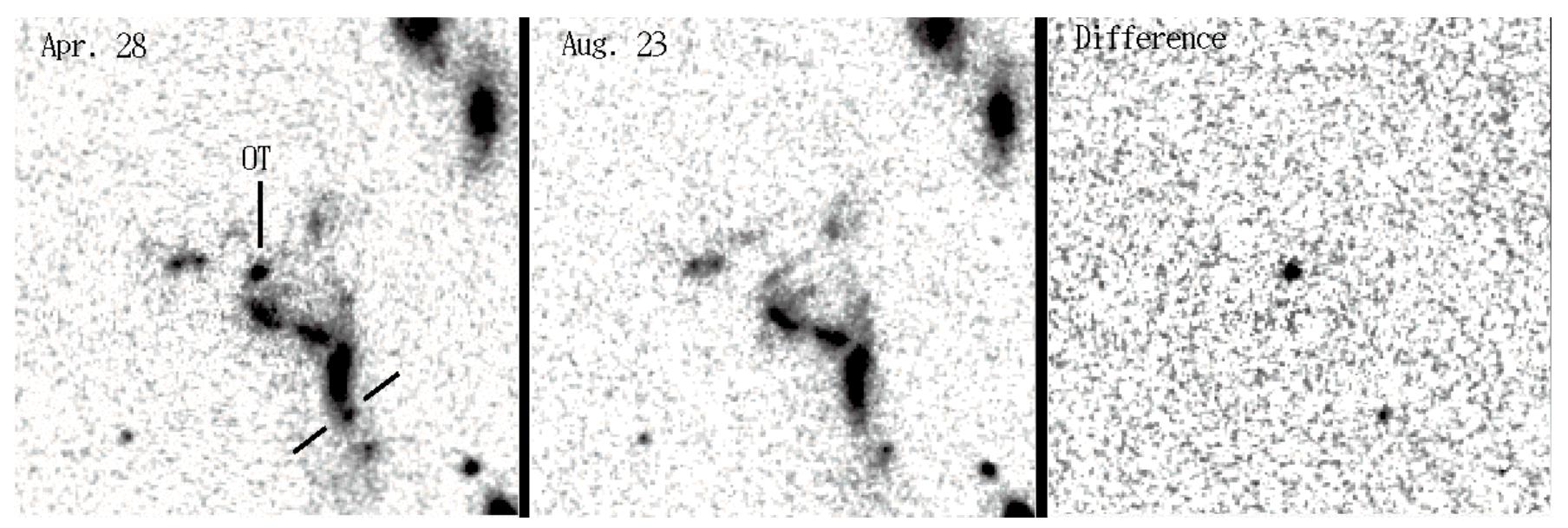

Fig. 3. HST-WFPC2 images of the OT field acquired with the F702W filter on April 28 (left panel) and August 23 (central panel), along with their difference (right panel). Besides the OT, located at the centre of the image, a second transient object (also indicated with tick marks in the April 28 image) is apparent $\sim 3^{\prime \prime}$ southwest of the OT itself. The images are about $10^{\prime \prime} \times 10^{\prime \prime}$ in size; North is at top, East is to the left.

indicating that no temporal break is apparent in our optical data set.

While the $J$-band light curve is reasonably fitted with a single power law with index compatible with that measured for the optical data, the $H$ and $K_{\mathrm{s}}$ data appear to decay with a shallower slope, $\alpha \sim 1.3$. Considering only the NIR data acquired in the first two days (which are presumably least contaminated by the host galaxy), we get an average decay slope $\alpha_{\mathrm{NIR}}=1.28 \pm 0.03$, substantially shallower than the one measured in the optical.

As already noted for this (Price et al. 2003) and for other, well sampled, afterglows (e.g., GRB010222: Masetti et al. 2001), a single or smoothly broken power-law fit does not formally give an acceptable fit to the VRI data. However, if we quadratically add a constant uncertainty of less than $3 \%$ to all $V R I$ points in our data set, the single power-law model gives an acceptable fit (with $\chi_{v}^{2} \sim 1$ ) for the same $\alpha$ obtained before. This formal discrepancy between the data and the model might be due to instrumental differences (e.g., the use of different detectors and different telescopes) or to intrinsic short time scale variations of the afterglow light curve (see e.g., Masetti et al. 2000; Holland et al. 2002; Jakobsson et al. 2002; Holland et al. 2003), possibly due to inhomogeneities of the circumburst medium (Wang \& Loeb 2000).

Between day 10 and 20 after the GRB, the OT decay flattens in all bands but more evidently in the RIJ bands, thus producing the red bump first noted by Price et al. (2003); thereafter, the flux decreases at a faster rate (Fig. 2). We fitted the data after day 20 to a single power law with index nearly independent of the wavelength and of average value $\alpha^{\prime}=1.85 \pm 0.15$. Interestingly, the $8.45 \mathrm{GHz}$ light curve of the radio afterglow (Berger et al. 2003) closely resembles this late-time optical behaviour.

Finally we note that the HST images in the filters F702W and $\mathrm{F} 814 \mathrm{~W}$ reveal the presence of a transient source located in the southern outskirts of galaxy "1" (as defined in Sect. 3.3 and Fig. 6), at coordinates (J2000) RA $=13^{\mathrm{h}} 58^{\mathrm{m}} 03^{\mathrm{s}} .01$; Dec $=-31^{\circ} 22^{\prime} 24^{\prime \prime} \cdot 7$ (J2000), with a conservative uncertainty of 0.1 on both. In Fig. 3 the position of this second transient is shown. This object faded between the end of April and the

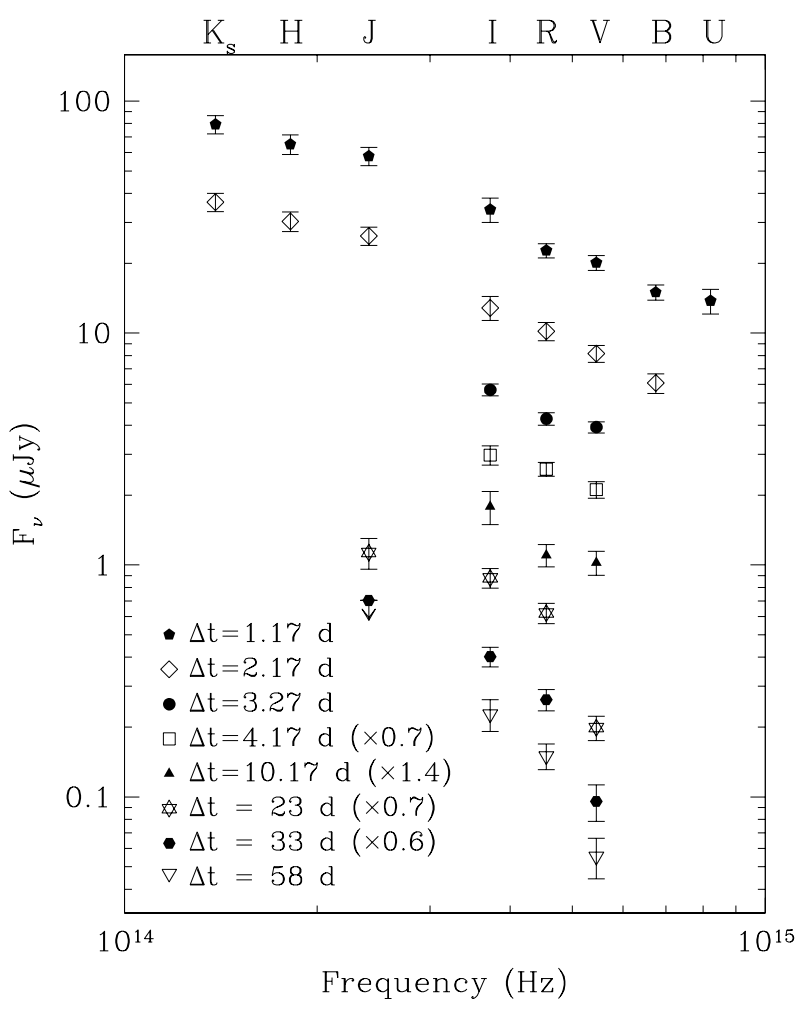

Fig. 4. Optical-NIR broad-band spectra of the GRB020405 afterglow at various epochs between $\sim 1$ and $\sim 60$ days after the GRB. Different symbol styles refer to different epochs. When needed, the broad-band spectra were rescaled in flux for clarity using the factors indicated in the figure. A spectral change located at $(2.5 \pm 0.6) \times 10^{14} \mathrm{~Hz}$ is apparent in the first two epochs. Assuming a broken power-law shape, optical and NIR spectral slopes are all consistent with being 1.3 and 0.65 , respectively, during the first $\sim 10$ days. In the last three epochs, the optical slope becomes 3.5 , thus significantly steeper, and a pronounced spectral curvature is apparent between the optical and NIR ranges.

beginning of June 2002, and is not detected in the August 2002 HST visit. It is also not detected in any of the F555W HST images. A more detailed description of this source will be given in a forthcoming paper. 

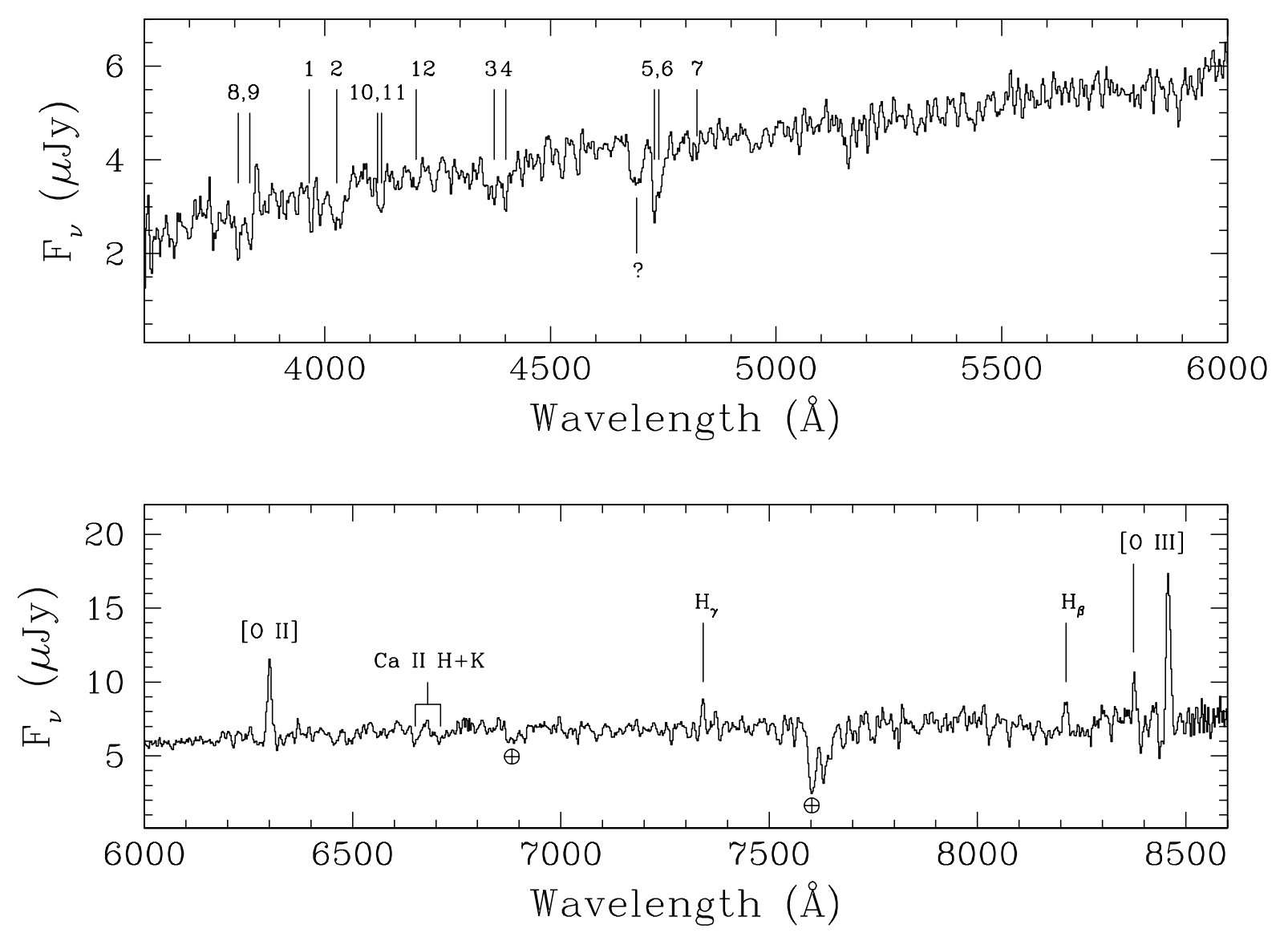

Fig. 5. Spectrum of the OT of GRB020405 obtained with VLT-Melipal plus FORS1 on 7 April 2002. The spectrum was smoothed with a Gaussian filter with $\sigma=3 \AA$ (i.e., comparable with the spectral resolution) and corrected for foreground Galactic absorption assuming $E(B-V)=0.055$. For clarity, we split it into two panels. Numbers mark the positions of the identified absorption lines as listed in Table 4, while the question mark indicates an unidentified line. Telluric absorption features at $6870 \AA$ and $7600 \AA$ are indicated with the symbol $\oplus$.

\subsection{Optical-NIR spectral flux distribution}

We have constructed optical-NIR broad-band spectra at 8 epochs spanning from $\sim 1$ to $\sim 60$ days after the GRB and for which we have sufficient optical and/or NIR coverage (Fig. 4). The data points were corrected for the Galactic absorption and converted into flux densities using the tables by Fukugita et al. (1995) for the optical and by Bersanelli et al. (1991) for the NIR. The spectra of the first two epochs were not corrected for the host contribution because this is known for the VRIJ bands only; however, given this was quite modest at those epochs (see Sect. 3.1), we simply added a 5\% error in quadrature to the uncertainties on the optical-NIR flux densities. The subsequent spectra were also corrected for the host galaxy contribution; the errors on the points include the uncertainty on the host photometry.

For the early epoch (within $\sim 10$ days after the GRB) broadband spectra, in the cases in which the data in different bands were not simultaneous, the flux density was interpolated to the reference epoch assuming the best-fit power-law decay (see Sect. 3.1). In the last three broad-band spectra the interpolations to a common epoch were computed using the power-law decay slope $\left(\alpha^{\prime}=1.85\right)$ determined from observations acquired after day 20 from the GRB.
Single power law $\left(F_{v} \propto v^{-\beta}\right)$ fits of the first two broadband spectra are unacceptable due to a significant deviation of the $J$-band points. Since we can firmly exclude instrumental effects or systematic errors in the $J$-band calibration, we fitted the spectra with broken power laws. The break is found to be located at $(2.5 \pm 0.6) \times 10^{14} \mathrm{~Hz}$, i.e., in the $J$-band, and the slopes are $\beta_{\mathrm{NIR}}=0.65 \pm 0.2$ and $\beta_{\mathrm{opt}}=1.3 \pm 0.2$, with no significant variability of these parameters between the two epochs. The optical slope agrees well, within the uncertainties, with those determined by Price et al. (2003) and Bersier et al. (2003).

The broad-band optical spectra of April 8.3, 9.2 and 15.2 UT were well fitted with single power laws, with spectral slopes $1.0 \pm 0.2,0.9 \pm 0.3$ and $1.35 \pm 0.5$, respectively; these are consistent with the optical slope measured in the first two epochs. The OT emission during the "red bump", measured 23, 33 , and 58 days after the GRB, is fitted by a power law of average spectral index $\beta=3.5 \pm 0.5$, in agreement with the findings of Price et al. (2003) and substantially steeper than at the earlier epochs. A remarkable NIR-to-optical spectral curvature is observed.

The optical and NIR colors of the OT of GRB020405 during the first 10 days of monitoring after the high-energy event fall in the loci populated by GRB afterglows in the color-color 
Table 4. List of emission and absorption lines identified in the VLT optical spectra of the GRB020405 OT acquired on April 6 and 7. The numbers in the leftmost column refer to the identifications shown in Fig. 5. The error on line positions is assumed to be $\pm 6 \AA$ for the spectrum of April 6 and $\pm 3 \AA$ for that of April 7, i.e., comparable with the corresponding spectral resolution (see text). For absorption lines we report the rest-frame $E W$, and for emission lines their intensity.

\begin{tabular}{|c|c|c|c|c|c|c|}
\hline $\begin{array}{l}\text { Line } \\
\text { number }\end{array}$ & $\begin{array}{c}\text { Observed } \\
\text { wavelength }(\AA)\end{array}$ & $\begin{array}{c}\text { Rest frame } \\
\text { wavelength }(\AA)\end{array}$ & Transition & Redshift & $\begin{array}{c}E W_{\text {rest }} \\
(\AA)\end{array}$ & $\begin{array}{c}\text { Flux } \\
\left(\times 10^{-17} \mathrm{erg} \mathrm{cm}^{-2} \mathrm{~s}^{-1}\right)\end{array}$ \\
\hline \multicolumn{7}{|c|}{6 April 2002} \\
\hline & 6302 & 3727 & [O II $]$ & $0.6908 \pm 0.0016$ & & \multirow{3}{*}{$9.5 \pm 1.5$} \\
\hline & 6647 & 3935 & Ca II K & $0.6893 \pm 0.0015$ & $1.2 \pm 0.3$ & \\
\hline & 6710 & 3969 & Ca II H & $0.6905 \pm 0.0015$ & $1.2 \pm 0.3$ & \\
\hline & 8227 & 4861 & $\mathrm{H}_{\beta}$ & $0.6923 \pm 0.0012$ & & $4.0 \pm 0.5$ \\
\hline & 8386 & 4959 & [O III] & $0.6910 \pm 0.0012$ & & $4.0 \pm 0.5$ \\
\hline & 8472 & 5007 & [O III] & $0.6920 \pm 0.0012$ & & $11.5 \pm 1.5$ \\
\hline & & Weighted mean & 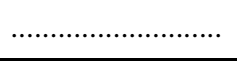 & $0.6910 \pm 0.0014$ & & \\
\hline \multicolumn{7}{|c|}{7 April 2002} \\
\hline 1 & 3965 & 2344 & $\mathrm{Fe}$ II & $0.6912 \pm 0.0013$ & $1.5 \pm 0.3$ & \\
\hline 2 & 4026 & $2374+2383$ & Fe II* & $0.6916 \pm 0.0013$ & $6.0 \pm 1.2$ & \\
\hline 3 & 4375 & 2587 & $\mathrm{Fe}$ II & $0.6915 \pm 0.0012$ & $0.8 \pm 0.3$ & \\
\hline 4 & 4401 & 2600 & Fe II & $0.6889 \pm 0.0012$ & $1.1 \pm 0.3$ & \\
\hline 5 & 4730 & 2796 & $\mathrm{Mg}$ II & $0.6913 \pm 0.0011$ & $2.7 \pm 0.4$ & \\
\hline 6 & 4740 & 2803 & $\mathrm{Mg}$ II & $0.6909 \pm 0.0011$ & $1.2 \pm 0.3$ & \\
\hline \multirow[t]{9}{*}{7} & 4825 & 2853 & Mg I & $0.6913 \pm 0.0011$ & $0.4 \pm 0.2^{* *}$ & \multirow{4}{*}{$8.5 \pm 1.0$} \\
\hline & 6302 & 3727 & [O II] & $0.6908 \pm 0.0008$ & & \\
\hline & 6650 & 3935 & Ca II K & $0.6900 \pm 0.0008$ & $1.2 \pm 0.3$ & \\
\hline & 6711 & 3969 & Ca II H & $0.6905 \pm 0.0008$ & $1.5 \pm 0.4$ & \\
\hline & 7342 & 4341 & $\mathrm{H}_{\gamma}$ & $0.6915 \pm 0.0007$ & & $1.3 \pm 0.5$ \\
\hline & 8213 & 4861 & $\mathrm{H}_{\beta}$ & $0.6895 \pm 0.0006$ & & $2.5 \pm 1.0$ \\
\hline & 8375 & 4959 & [O III] & $0.6888 \pm 0.0006$ & & $3.0 \pm 1.0$ \\
\hline & \multirow[t]{2}{*}{8460} & 5007 & [O III] & $0.6897 \pm 0.0006$ & & $11.0 \pm 2.0$ \\
\hline & & Weighted mean & 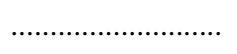 & $0.6905 \pm 0.0010$ & & \\
\hline 8 & 3808 & 2587 & Fe II & $0.4720 \pm 0.0012$ & $2.4 \pm 0.4$ & \\
\hline 9 & 3834 & 2600 & $\mathrm{Fe}$ II & $0.4714 \pm 0.0012$ & $2.5 \pm 0.4$ & \\
\hline 10 & 4117 & 2796 & $\mathrm{Mg}$ II & $0.4723 \pm 0.0011$ & $1.1 \pm 0.3$ & \\
\hline 11 & 4126 & 2803 & $\mathrm{Mg}$ II & $0.4717 \pm 0.0011$ & $1.1 \pm 0.3$ & \\
\hline \multirow[t]{2}{*}{12} & 4202 & 2853 & Mg I & $0.4729 \pm 0.0011$ & $0.4 \pm 0.3^{* *}$ & \\
\hline & & Weighted mean & ................... & $0.4720 \pm 0.0011$ & & \\
\hline
\end{tabular}

*Blend of two Fe II lines. The restframe centroid is roughly estimated to be at $2380 \AA$.

${ }^{* *}$ This identification is only tentative; it is however included in the list as it coincides with the redshift of this absorption system.

diagrams as illustrated by Šimon et al. (2001) and by Gorosabel et al. (2002).

\subsection{Optical spectra}

The continuum slopes measured for the VLT spectra of April 6 and 7 are consistent with the results of our broad-band spectral analysis reported in the previous Subsection.

In the OT spectra of April 6 and 7 we detected a number of emission lines which we identified with the forbidden transitions of [O II] $\lambda 3727,[\mathrm{O}$ III] $\lambda \lambda 4959,5007$, and with the $\mathrm{H}_{\beta}$ line at an average redshift of $0.6908 \pm 0.0017$. In the spectrum of April 7 (Fig. 5) we also possibly detect $\mathrm{H}_{\gamma}$ in emission. Our redshift measurement is confirmed by the detection of several absorption features blueward of $5000 \AA$ in the April 7 spectrum. These absorptions are due to rest-frame ultraviolet (UV) metallic lines usually present in OT spectra and, more generally, in the spectra of high- $z$ objects (see, e.g., Savaglio et al. 2003 and references therein). In this wavelength range we also detect an absorption system at a lower average redshift $z=0.4720 \pm 0.0011$.

Our line fitting, performed with the SPLOT task within IRAF, assumes a Gaussian profile for both emission and absorption lines. A conservative error of $6 \AA$ and $3 \AA$, comparable with the spectral resolution, is associated with line wavelength measurements in the spectra of April 6 and 7, respectively. In Table 4 are reported, for each line detected in the two spectroscopic runs, the identification along with the observed and rest-frame wavelengths and the associated redshift. No significant difference in the emission and absorption line redshifts is found in either observation for the features at $z=0.691$. In Table 4 we also list the equivalent widths $(E W s)$ of the 


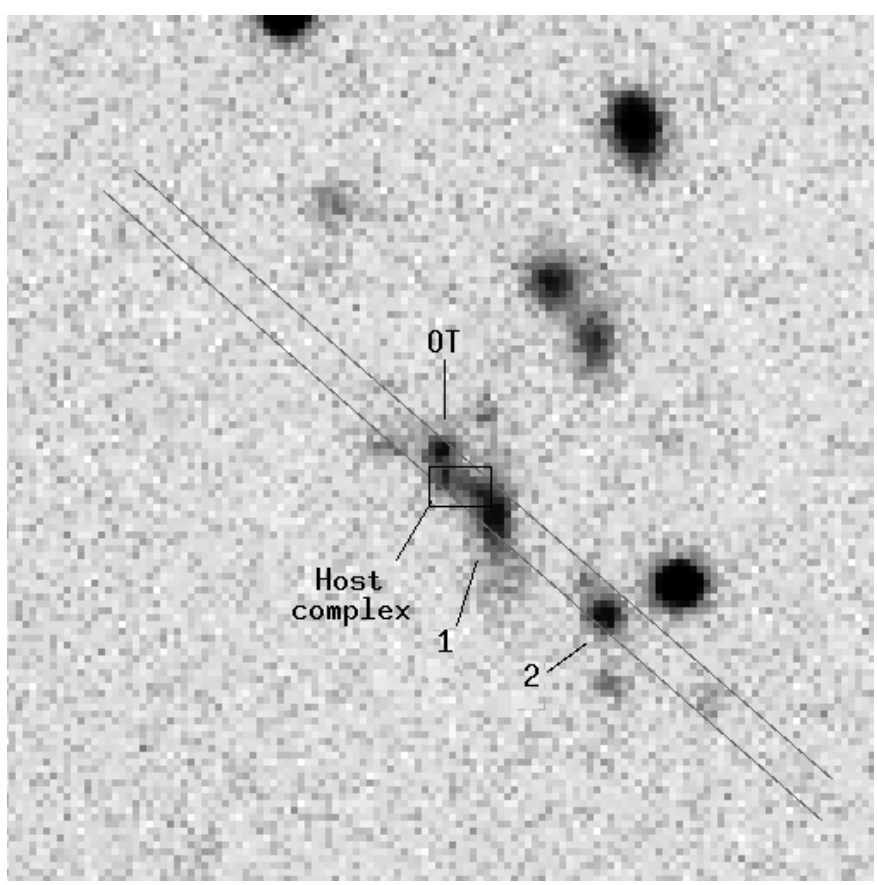

Fig. 6. VLT-Melipal (+FORS1) R-band image of the GRB020405 field acquired on 15 April 2002. The host galaxy of the GRB and galaxies "1" and " 2 " described in the text are indicated. The field size is about $25^{\prime \prime} \times 25^{\prime \prime}$; North is at top, East is to the left. The diagonal solid lines indicate the slit position and width of the VLT spectrum obtained on 7 April 2002.

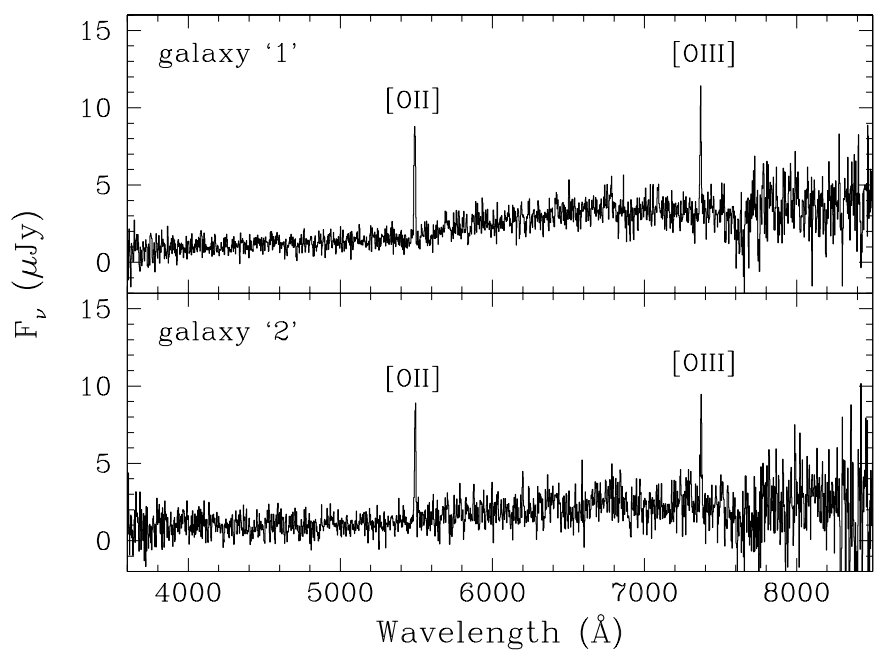

Fig. 7. Spectra of galaxies "1" (upper panel) and "2" (lower panel) lying southwest of the OT (see Fig. 6) acquired on April 7 with VLTMelipal plus FORS1. The [O II] $\lambda 3727$ and [O III] $\lambda 5007$ emission lines, at a redshift $z=0.472$, are indicated.

identified lines computed in the absorber rest frame, i.e., dividing the measured value by the factor $(1+z)$. The errors on the $E W \mathrm{~s}$ are computed by assuming for the spectral continuum in proximity of each line the values corresponding to its $1-\sigma$ lower and upper bounds. We did not find any significant variation in the emission line fluxes between the two nights, as expected if the line production sites are gas-rich and star-forming regions of the GRB host galaxy. The $E W$ s of the only absorption lines detected in both nights (those of Ca II) do not vary
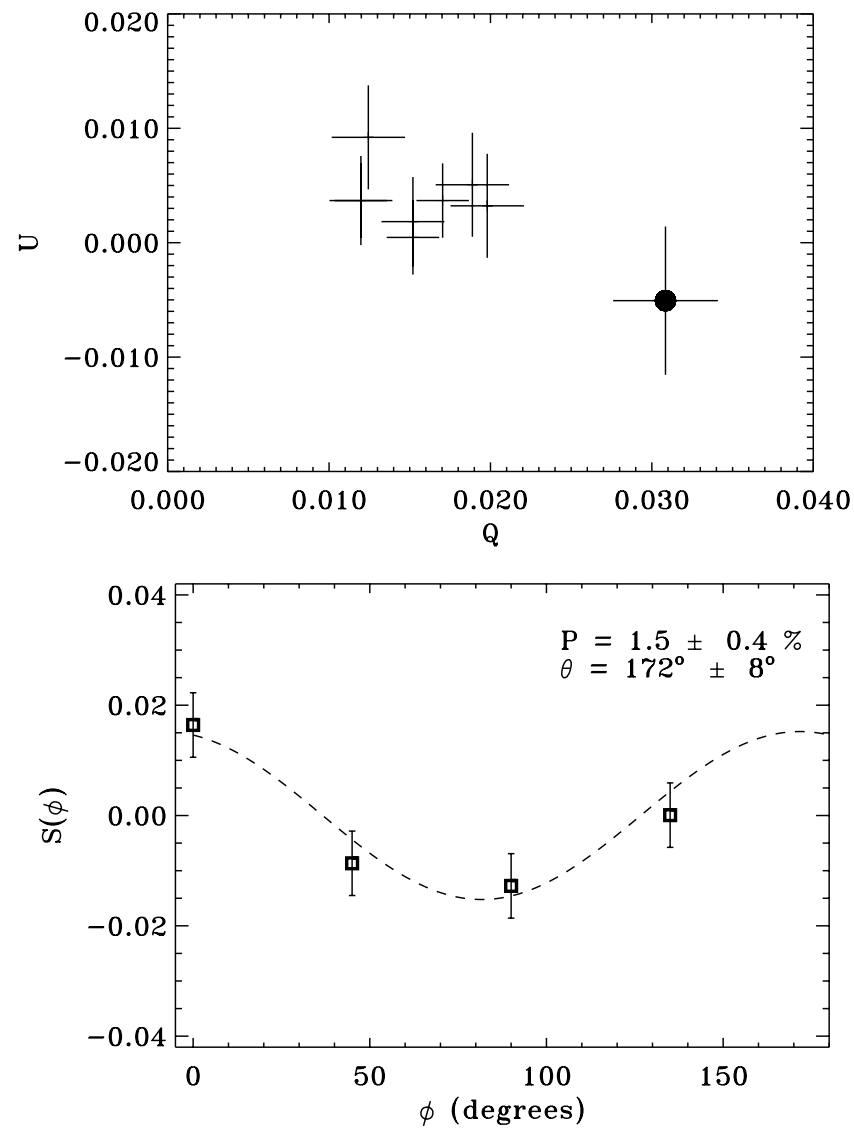

Fig. 8. (Upper panel) positions of field stars and of the OT (marked with a filled dot) in the plane of the $U$ and $Q$ parameters not corrected for spurious (instrumental plus field) polarization. The OT is clearly separated from the region occupied by the field stars: this indicates that it has net intrinsic polarization. (Lower panel) cosine fit of the $R$ band polarimetric data of April 6. The best fit yields $P=1.5 \pm 0.4 \%$ and $\theta=172^{\circ} \pm 8^{\circ}$.

significantly. We also detected a broad absorption feature (the observer's frame $E W$ is $5.6 \pm 0.5 \AA$ ) at $4691 \AA$ which cannot be identified with any known line among those typically detected in OT spectra at either of the two detected redshifts. This feature is indicated with a question mark in Fig. 5.

The slit orientation used for the spectroscopic observations acquired on April 7 also allowed us to acquire spectra of the nearby galaxy first noted by Hjorth et al. (2002). Figure 6 reports a sketch of the setup with which the VLT spectrum of April 7 was obtained: the $1^{\prime \prime}$-wide slit was rotated by $40^{\circ}$ towards East with respect to the N-S direction in order to acquire also the spectrum of the galaxy at 2 " southwest of the OT, marked as " 1 " in Fig. 6 (see also Hjorth et al. 2002). The slit also included a galaxy (indicated as " 2 " in the same figure) located $\sim 6^{\prime \prime}$ southwest of the OT. The spectra of these two galaxies are reported in Fig. 7.

From the detection of [O II] $\lambda 3727$ and [O III] $\lambda 5007$ emission lines in their spectra, galaxies " 1 " and " 2 , 'appear to be both at redshift $z=0.472 \pm 0.001$, substantially lower than that of the GRB. This is the same redshift of one of the absorption systems detected in the OT spectrum. Therefore, galaxy " 1 " is most probably responsible for that absorption 
system. Moreover, galaxy " 1 " cannot be interacting with the GRB host. This result overrides that reported in Masetti et al. (2002b): the preliminary analysis of the spectrum of galaxy " 1 " led to an incorrect result because the contamination by the host galaxy of the GRB, at $z=0.691$, had not been properly removed.

\subsection{Polarimetry}

In order to evaluate the Stokes $Q$ and $U$ parameters of the OT emission, and thence its polarization percentage $P$ and position angle $\theta$, we applied the method described by di Serego Alighieri (1997). Here $P$ and $\theta$ are obtained by using the relation $S(\phi)=P \cos 2(\theta-\phi)$, in which $S$ depends on the ratio between the fluxes of ordinary and extraordinary components of the incident beam, and $\phi$ corresponds to the prism rotation angle. The values of $P$ and $\theta$ are calculated by fitting the above relation to the measurements obtained in correspondence of each rotation angle. Moreover, using this formalism, one obtains the two Stokes parameters $Q$ and $U$ from the values of $S\left(0^{\circ}\right)$ and $S\left(45^{\circ}\right)$, respectively. The intrinsic nature of the OT $R$-band polarization is supported by the relative location of the OT with respect to that of field stars in the $U$ vs. $Q$ plot (Fig. 8, upper panel).

After correcting for spurious field polarization, we found $Q_{\mathrm{OT}}=0.016 \pm 0.006$ and $U_{\mathrm{OT}}=-0.009 \pm 0.006$. The fit of the data with the relation described above (see Fig. 8, lower panel) yielded for the OT a linear polarization $P_{\mathrm{OT}}=1.5 \pm 0.4 \%$ and a polarization angle $\theta_{\mathrm{OT}}=172^{\circ} \pm 8^{\circ}$, corrected for the polarization bias (Wardle \& Kronberg 1974). This latter correction is introduced because $P$ is a positive definite quantity, and thus at low $\mathrm{S} / \mathrm{N}$ polarization levels the distribution function of $P$ is no longer normal but becomes skewed, which causes an overestimate of the real value of $P$ (Simmons \& Stewart 1985).

The use of standard polarization equations (e.g., Ramaprakash 1998) leads to results which are in good agreement, within the uncertainties, with those obtained from the method described above. In our treatment, we implicitly assumed that the OT polarization degree and angle did not vary across the four exposures based on the fact that the total flux varies only by $0.025 \mathrm{mag}$ in this time interval.

The spurious polarization contribution due to the galactic ISM and produced by dust grains located along the line of sight (preferentially aligned in one direction) depends on the $E(B-$ $V$ ) color excess according to the empirical relation (Hiltner 1956; Serkowski et al. 1975) $P_{\mathrm{ISM}}(\%)=9.0 \times E(B-V)$. In this case $E(B-V)=0.055$, as mentioned above, so the total ISM-induced polarization is $0.5 \%$. The fraction of this due to the close Galactic ISM has been taken into account and corrected by normalizing the OT polarization measurement to several field stars, as described in Sect. 2.3. Indeed, we find that the overall spurious polarization of the field stars is $P_{\mathrm{sp}}=0.5 \pm 0.1 \%$. This means that it is entirely due to the Galactic ISM, which further confirms that the instrumental polarization is negligible. We do not expect that the far (Galactic Halo) ISM contributes significantly to the OT polarization. The contribution of the host galaxy to the total (OT+host) light is just few percent; therefore we do not expect that the host emission contaminated significantly our polarization measurement (see also Covino et al. 2003). Concerning possible additional local dust absorption within the host (see the caveats of Covino et al. 2003 about this issue), we will show in Sect. 4.2 that we do not find evidence for this, based on the analysis of the emission of the GRB020405 afterglow. Therefore we tend to conclude that the measured OT polarization is intrinsic.

\section{Discussion}

\subsection{Light curves: SN vs. shock re-energization}

The optical light curves of the GRB020405 afterglow are consistent with a single power-law decay of temporal index $\alpha=$ $1.54 \pm 0.06$ between 1 and 10 days after the prompt event, with no temporal break. In the fireball scenario, this points to a nearly spherically symmetric expansion (Sari et al. 1998), as also suggested by our fits to the broad-band spectra of the afterglow (see Sect. 4.2). Our temporal slope is different than those obtained by Bersier et al. (2003) and Price et al. (2003), likely due to the longer time baseline of our sampling and to the lower host galaxy contribution resulting from our analysis. For the NIR light curves we find a slightly shallower temporal decay, $\alpha=1.28 \pm 0.03$.

The red bump detected $\sim 20$ days after the GRB has been modeled by Price et al. (2003) with an emerging SN which is 0.5 mag dimmer than the Type Ic SN1998bw $(z=0.0085)$ - considered the "hypernova" prototype (Paczyński 1998; Galama et al. 1998; Patat et al. 2001) - exploded simultaneously with the GRB at its redshift. Similarly, Dado et al. (2002) modeled the light curve with a SN akin to $1998 \mathrm{bw}$, but with the addition of intrinsic extinction. We have also applied a composite power-law-plus-SN fit to our VRIJ light curves, which extend over a longer time interval than that considered by the above authors. We first tried a fit with SN1998bw as a template, redshifted to $z=0.691$, and then we used the light curves of another Type Ic SN, 2002ap (Nakano et al. 2002; Gal-Yam et al. 2002b; Pandey et al. 2003), located in M74 (8 Mpc) which has also exhibited some hypernova characteristics (Mazzali et al. 2002; see however Berger et al. 2002). SN2002ap showed a faster time evolution after maximum with respect to that of SN1998bw, and therefore it could possibly better describe the observed post-flattening OT decay. To construct light curves at the effective wavelengths of the GRB020405 afterglow observations we interpolated the light curves of the two model $\mathrm{SNe}$ or extrapolated to the rest-frame UV range. In order to optimize the fit, the SN1998bw and SN2002ap temporal profiles were dimmed by 0.6 mag and brightened by $1.3 \mathrm{mag}$, respectively.

In Fig. 9 are reported the power-law+SN fits for the case in which the SN2002ap was used as a template. Figure 10 shows the fit with a power law plus SN1998bw in the $R$-band only. Either using SN1998bw or SN2002ap as a template, the fit is not satisfactory in the $V$-band. This can be expected, since this wavelength range corresponds to the near-UV at the GRB redshift, where SNe suffer severe intrinsic extinction in the envelope. In the other bands, the fit is marginally satisfactory for 

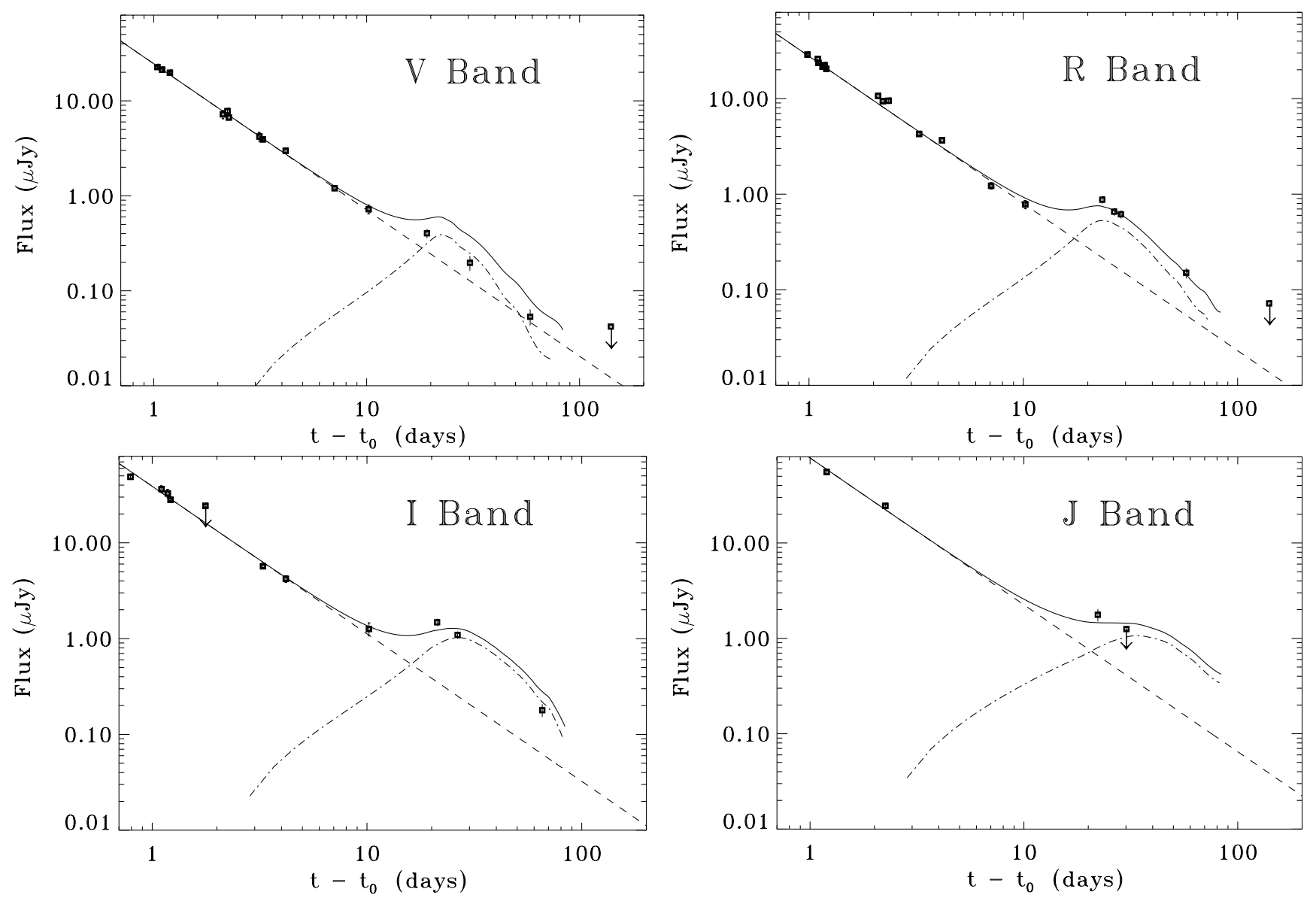

Fig. 9. $V$ (upper left), $R$ (upper right), $I$ (lower left) and $J$ (lower right) light curves of the GRB020405 afterglow; $t_{0}$ indicates the time of the GRB onset. The data are corrected for the host galaxy contribution and for Galactic absorption, and fitted with a power law with $\alpha=1.54$ (dashed line) plus a SN2002ap at $z=0.691$ (dot-dashed line) brightened by 1.3 mag with respect to the original one. The solid line represents the best-fit model. In these fits the SN was considered as occurred simultaneously with the GRB. Light curves of the SN2002ap template end at $\sim 80$ days after the explosion because after that date they are poorly sampled.

both SN templates. Allowing for a positive or negative time lag between the SN and GRB explosions does not produce any significant improvement in the fit.

Alternatively, the afterglow temporal variability may be reproduced by both density and energy variations (e.g., Nakar et al. 2003). Specifically, the observed bump may be interpreted within the scenario of shell collisions proposed by Kumar \& Piran (2000), in which a slower shell runs into a faster but decelerating shell and re-energizes it. The amplitude of the bump depends on the ratio of the energy amounts carried by the two shells. The optical light curves of GRB020405 exhibit a larger temporal slope $\left(\alpha^{\prime}=1.85 \pm 0.15\right)$ after the bump than before it $(\alpha=1.54 \pm 0.06)$, which would imply also a change in the electron energy distribution shape, an effect which is not predicted by Kumar \& Piran (2000). While this circumstance may limit the applicability of their picture to our observations, a similar but more refined model could be consistent with them. Our light curves could be also qualitatively reproduced by an analogous scenario proposed by Ramirez-Ruiz et al. (2001), in which light variations are caused by the interaction of the blast wave with the density bumps in a pre-ejected stellar wind. However, this interpretation would not be self-consistent, since the hydrodynamics of the GRB020405 afterglow points to a homogeneous, rather than to a windy, circumburst medium (see below).

Other views are offered by the model developed by Beloborodov (2003), in which the fireball is interacting with a trailing neutron shell, or by that in which a SN remnant, located around the GRB progenitor and excited by the GRB itself, cools down on time scales of weeks (Dermer 2002). Unfortunately, no thorough quantitative descriptions of the effects induced by these interactions on the spectral and temporal behaviour of OTs are available at the moment.

The dust echo model (Esin \& Blandford 2000; Reichart 2001) seems instead to be a less viable interpretation for the emission excess because it predicts bluer spectra at the time of the bump compared with those displayed by the early-time observations assuming scattered light dust echo; this dust echo emission is ruled out by the data. This model however, in case of thermal dust echo, also foresees a possible spectral peak in the observer's frame far-IR, which we cannot test. However, the increase in the radio flux noticed by Berger et al. (2003) around day 20 after the GRB may be consistent with this explanation. The microlensing interpretation (e.g., Loeb \& Perna 1998) of the phenomenon is instead unlikely, as any variation is expected to be achromatic in this case. 
The temporal decay steepening seen in the optical at day $\sim 20$ after the GRB $\left(\alpha^{\prime}=1.85\right)$ might also be interpreted as a transition to a non-relativistic regime (Dai \& Lu 1999) for the same electron distribution shape derived below in Sect. 4.2. However, in this case we would not expect significant spectral steepening after the transition, as opposed to what is observed.

\subsection{The broad-band spectrum}

Mirabal et al. (2003) propose a wind model for the GRB020405 afterglow expansion: the blast wave propagates in a medium enriched before the GRB explosion by a wind ejected from a Wolf-Rayet star. Under the hypothesis that the cooling frequency is higher than the X-ray energies at the epoch of their Chandra observations, those authors find, based only on the $\mathrm{X}$-ray data, an electron distribution slope of $p=2.7$. However, the $X$-ray spectral shape, with a spectral index $\beta_{X}=0.7 \pm 0.2$, is significantly flatter than the one we derive in the optical, $\beta_{\text {opt }}=1.3 \pm 0.2$. If one allows for some intrinsic extinction within the GRB host galaxy, one may recover the agreement between the optical and X-ray spectral indices; however, in this case it would be difficult to explain why the temporal decline rate is different at the NIR, optical and X-ray frequencies (Chevalier \& Li 2000). If instead no intrinsic extinction is invoked, the flatness of the X-ray spectrum makes it impossible to reconcile it with synchrotron emission, predicted by the standard fireball scenario to be produced as a consequence of the interaction between the external shock and the interstellar medium (Sari et al. 1998) or the circumburst medium enriched by a pre-ejected stellar wind (Chevalier \& Li 2000). Therefore, we favor an interpretation of the $\mathrm{X}$-rays as Inverse Compton emission, as also proposed by Mirabal et al. (2003), and as in the case of GRB000926 (Harrison et al. 2001). Since the Xray spectral slope is consistent, within the errors, with the NIR slope, the same population of relativistic electrons may have produced the NIR photons via synchrotron radiation and the $\mathrm{X}$-rays via Inverse Compton scattering off afterglow radiation (synchrotron self-Compton).

The optical and NIR data are inconsistent with a wind afterglow model (Chevalier \& Li 2000): the spectral break between the NIR and optical bands (Fig. 4) could not correspond to the synchrotron injection break $\left(v_{\mathrm{m}}\right)$, since then the observed NIR slope would be expected to be much flatter than observed; therefore, this break must be due to the synchrotron cooling frequency $\left(v_{\mathrm{c}}\right)$. However, in this case the NIR temporal decline should be faster than seen in optical, contrary to what is observed. Assuming instead an isotropic and adiabatic expansion in an external medium of constant density ("homogeneous" model; Sari et al. 1998) and a slow cooling regime, the spectral slopes bluewards and redwards of the cooling frequency $v_{\mathrm{c}} \simeq 2.5 \times 10^{14} \mathrm{~Hz}$ imply an electron energy distribution index $p=2.5 \pm 0.3$. This is consistent with the (better constrained) value, $p=2.7 \pm 0.1$, obtained from the NIR and optical decay rates under the assumptions of the homogeneous model with no need for additional local absorption in the host.

From this, and from the above considerations on the broadband X-ray-optical-NIR spectral flux distribution, we conclude

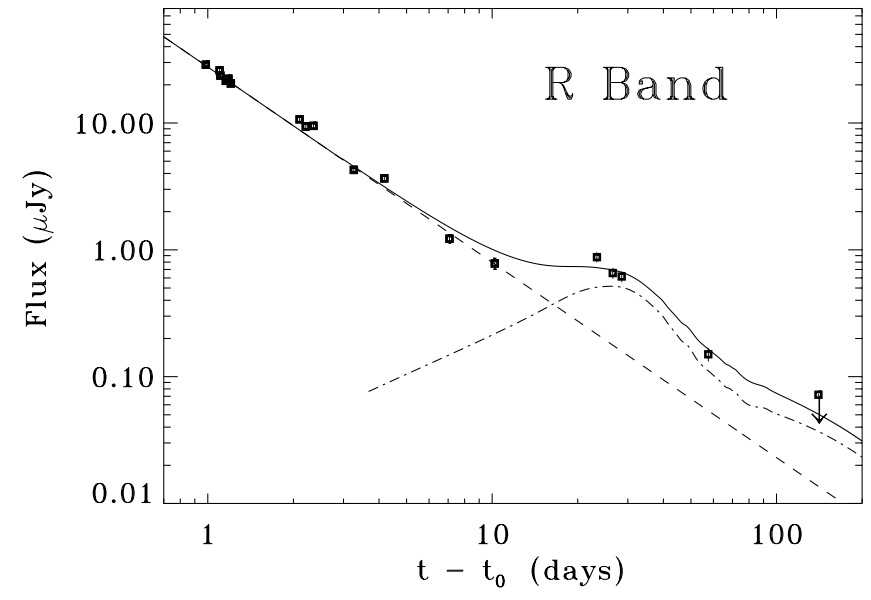

Fig. 10. Same as the upper right panel of Fig. 9, but with SN1998bw as a template, dimmed by $0.6 \mathrm{mag}$.

that the fireball emission is affected by negligible wavelengthdependent extinction associated with the host galaxy. Similarly, the OT detection in the $U$-band ( $\lambda_{\text {restframe }} \sim 2100 \AA$ ) suggests, in a model-independent way, negligible intrinsic absorption.

We note that a collimated fireball before the jet break (Sari et al. 1999; Rhoads 1999) and an isotropic fireball (Sari et al. 1998) are described in equivalent ways by the standard model and that they cannot be discerned by the temporal and spectral indices. If the bump observed in the light curves at day $\sim 20$ is due to an emission component independent from the afterglow (i.e., a SN), our data cannot rule out a steepening of the afterglow light curve, related to a collimation break, occurring more than $\sim 10$ days after the GRB. According to Sari et al. (1999), this would imply a lower limit to the jet opening angle of $\Theta>14^{\circ}$.

The conclusion that the GRB020405 fireball expanded in a homogeneous medium was independently reached by Berger et al. (2003) from observations of the radio afterglow. However, these authors also suggest the presence of a jet break occurred $\approx 1$ day after the GRB. While our optical-NIR light curve coverage does not allow us to confirm their finding, this interpretation would imply that we monitored the counterpart decay during the post-break phase of a collimated fireball. Following Sari et al. (1999), this would require that $p \equiv \alpha \sim 1.5$, which is inconsistent with our spectral results.

\subsection{Optical polarization}

Our $R$-band polarization measurement is in line with the detections and upper limits so far obtained for GRB optical afterglows (Hjorth et al. 1999; Wijers et al. 1999; Covino et al. 1999; Rol et al. 2000; Björnsson et al. 2002; Covino et al. 2002c; Covino et al. 2002d; Covino et al. 2002e; Rol et al. 2003).

Jet geometries are thought to produce observable polarization (Sari 1999; Ghisellini \& Lazzati 1999), however, no indication of a jet structure is derived from the light curve analysis for the GRB020405 afterglow (see Sects. 3.1 and 4.1). These characteristics, i.e., the presence of detectable 
polarization and the absence of a light curve steepening (at least in the first 10 days), are reminiscent of GRB990712 optical afterglow (Rol et al. 2000; Sahu et al. 2000), although a jet break has been suggested by Björnsson et al. (2001) for the latter.

Our values of $P$ and $\theta$ are consistent with those measured for the present afterglow by Covino et al. (2003) in the $V$ band $\sim 2$ and $\sim 3$ days after the GRB. However, our polarization percentage measurement is at variance with that obtained by Bersier et al. (2003) from $V$-band observations acquired nearly simultaneously with ours. This cannot be explained by the contribution from the underlying host galaxy, because (as we remarked in Sect. 3) this is negligible at the time of our polarimetric observation. Also, a strong dependence of the polarization value on the wavelength is unlikely (Rybicki \& Lightman 1979) and, indeed, recent optical spectropolarimetric observations of the afterglows of GRB020813 (Barth et al. 2003) and GRB021004 (Wang et al. 2003) show no substantial variations of $P$ across the continuum redwards of $4000 \AA$.

For a spherically symmetric fireball, the net polarization should vanish if the magnetic field is ordered exactly perpendicular or parallel with respect to the expanding shock front (e.g., Waxman 1997). Therefore, rapidly variable effects removing the symmetry should be considered in order to explain the different polarization values measured by us and by Bersier et al. (2003), like turbulence or microlensing (Medvedev \& Loeb 1999). In these cases substantial variations of $\theta$ would also be expected (see Rol et al. 2000 and references therein), but they are not observed. Therefore, if real, this fast variation of $P$ lacks a satisfactory interpretation.

Thus, as also pointed out by Rol et al. (2000) for GRB990712, no current polarization model adequately explains the entire optical polarimetry data set for the GRB020405 afterglow, that is, the strong variability of $P$ and the simultaneous constancy of $\theta$ when no break is seen in the optical light curve during the polarimetric monitoring (see however Björnsson \& Lindfors 2000). We independently analyzed the polarimetric data of Bersier et al. (2003) and, by applying our PSF-fitting photometric procedure, which differs from that based on the ISIS2 package (Alard 2000) used by Bersier et al. (2003), we obtain a 3- $\sigma$ upper limit on the $V$ band polarization of $36 \%$. This value is higher than, but still consistent with the measurement and upper limit obtained by the above authors.

\subsection{The host galaxy and the intervening absorption}

The ground-based and HST imaging reveal that the GRB020405 OT is located in a complex field (see Figs. 3 and 6). Emission and absorption lines detected on the optical spectra of the OT indicate a redshift of $z=0.691$, confirming our preliminary analysis (Masetti et al. 2002a) and the independent spectroscopic results by Price et al. (2003). An absorption system at the lower redshift of $z=0.472$ is also detected in the OT spectrum acquired on April 7 (see Fig. 5), and we attribute it to galaxy "1" (Fig. 6), for which we detect [O II] and [O III] in emission at the same redshift in the VLT spectrum. At this redshift the angular separation $\left(\sim 2^{\prime \prime}\right)$ between galaxy " 1 " and the OT corresponds to $\sim 13$ proper kpc, consistent with the halo of galaxy " 1 " being responsible for the absorption system at $z=0.472$ detected in the OT spectrum. For galaxy "2", located at $\sim 6^{\prime \prime}$ from the OT (Fig. 6), we also measured a redshift $z=0.472$, therefore the two galaxies probably belong to a foreground association or cluster. Galaxy " 2 " may also be responsible for the absorption system, although its larger angular distance from the OT makes it a less probable candidate than galaxy " 1 " for the absorber. The presence of intervening absorption systems is not uncommon in the OT spectra (e.g., Metzger et al. 1997; Masetti et al. 2001; Barth et al. 2003; Møller et al. 2002). However, the present case is the first for which the intervening absorber has been identified with imaging and spectroscopy.

The flux of the [O II] $\lambda 3727$ can be used to determine the star formation rate (SFR) of the GRB020405 host galaxy. Applying Eq. (3) of Kennicutt (1998), we determine a SFR of $\sim 3 M_{\odot} \mathrm{yr}^{-1}$, consistent with the findings of Price et al. (2003). As noted by these authors, this is actually a lower limit to the SFR as no correction for the unknown local absorption has been determined.

The detection of [O II], [O III] and $\mathrm{H}_{\beta}$ emission lines in the spectrum also allowed us to infer the oxygen abundance in the GRB host. By computing the parameter $R_{23}$, defined by Kobulnicky et al. (1999) as the ratio between the sum of the oxygen forbidden line fluxes and the $\mathrm{H}_{\beta}$ line flux, we find $R_{23}=6.5 \pm 2$ which implies, from Fig. 8 of Kobulnicky et al. (1999), that $12+\log (\mathrm{O} / \mathrm{H})=8.0 \pm 0.25$. This suggests that the GRB020405 host has an oxygen abundance lower (around one eighth) than solar; also, this metallicity yield is similar to those found for other GRB hosts (e.g., Price et al. 2002c).

\section{Conclusions}

The most important results of our ground-based observing campaign on the GRB020405 afterglow can be summarized as follows:

- We have reported the first detection of the NIR afterglow of this GRB: the OT is clearly detected in $J H K_{\mathrm{s}}$ bands.

- Thanks to our accurate method of host subtraction we find that, between 1 and 10 days after the GRB, the decay of the afterglow light curves is consistent with a single power law. Therefore we model the afterglow emission as synchrotron radiation in a fireball expanding in a homogeneous medium and within a jet whose opening angle is not smaller than $14^{\circ}$. At X-ray frequencies, the contribution of synchrotron self-Compton radiation may dominate.

- The addition of late-time (20-150 days after the GRB) HST points and VLT $J$-band observations indicates the presence of a red bump in the VRIJ light curves around 20 days after the GRB. This bump can be modeled with a SN. Alternatively, HST and late-time $J$-band data can be modeled by using a power law with decay index steeper than that of the early decline phase $\left(\alpha^{\prime}=1.85 \pm 0.15\right)$. This can be explained as due to a late shell collision in the fireball.

- A $R$-band polarimetry measure shows that the afterglow is polarized, with $P=1.5 \pm 0.4 \%$ and $\theta=172^{\circ} \pm 8^{\circ}$. 
No quantitative model can explain the entire polarization data set for this OT.

- The spectroscopic detection of emission lines indicates that the GRB is located at redshift $z=0.691$. Two metallic absorption systems, at $z=0.691$ and at $z=0.472$, are also detected in the afterglow optical spectrum. This latter redshift coincides with that of a galaxy complex angularly close $\left(2^{\prime \prime}\right)$ to the host of GRB020405, indicating that the absorption system most likely originated in this complex. For the first time, the galaxy responsible for an intervening absorption line system in the spectrum of an OT is directly detected.

- The host of GRB020405 appears to be a galaxy with moderate SFR $\left(\sim 3 M_{\odot} \mathrm{yr}^{-1}\right)$ and with subsolar metallicity.

- While monitoring the late phases of the afterglow decay, HST revealed the presence of a further variable object located $\sim 3^{\prime \prime}$ from the OT, in the southern outskirts of galaxy " 1 ". While its nature is still to be investigated, its presence underscores the need for a careful validation of the variable candidates detected in GRB error boxes as counterparts of the high energy events.

Our results point to the need of further systematic NIR/optical campaigns of GRB afterglows, coordinated with higher energy monitorings, to better constrain the emission mechanisms and the environmental characteristics of GRBs and of their lower energy counterparts.

Acknowledgements. We are grateful to the referee, Dr. E. Le Floc'h, for several useful comments which helped us to improve the paper. We thank R. Silvotti, D. Stephens and D. Thomas for having kindly allowed us to acquire some of the data presented here during their observational time. We also thank the ESO staff astronomers M. Billeres (La Silla) and H. Böhnhardt, R. Cabanac, A. Kaufer, R. Johnson and A. Jaunsen (Paranal) as well as J. Licandro (TNG), T. Augusteijn and R. Greimel (WHT and JKT) for their help and efforts in obtaining the observations presented in this paper. The TNG data presented here were acquired in the framework of the Italian GRB Collaboration at TNG. Paul Price is thanked for having swiftly provided us the position of the OT soon after its discovery, as well as the June 2002 HST data prior to their public release. We acknowledge Scott Barthelmy for maintaining the GRB Coordinates Network $(\mathrm{GCN})$ and BACODINE services. This research has made use of NASA's Astrophysics Data System and of the Multimission Archive at the Space Telescope Science Institute (MAST). This work was supported by the Danish Natural Science Research Council (SNF). J. M. Castro Cerón acknowledges the receipt of a FPI doctoral fellowship from Spain's Ministerio de Ciencia y Tecnología.

\section{References}

Alard, C. 2000, A\&AS, 144, 363

Barth, A. J., Sari, R., Cohen, M. H., et al. 2003, ApJ, 584, L47

Beloborodov, A. M. 2003, ApJ, 585, L19

Berger, E. J., Kulkarni, S. R., \& Chevalier, R. A. 2002, ApJ, 577, L5

Berger, E. J., Soderberg, A. M., Frail, D. A., \& Kulkarni, S. R. 2003, ApJ, 587, L5

Bersanelli, M., Bouchet, P., \& Falomo, R. 1991, A\&A, 252, 854

Bersier, D., McLeod, B., Garnavich, P. M., et al. 2003, ApJ, 583, L63

Beuermann, K., Hessman, F. V., Reinsch, K., et al. 1999, A\&A, 352, L26
Biretta, J., et al. 2002, WFPC2 Instument Handbook, Version 7.0 (Baltimore, STScI); see also http://www.stsci.edu/documents/dhb/web/ c32_wfpc2dataanal. fm1.html

Björnsson, G., \& Lindfors, E. 2000, ApJ, 541, L55

Björnsson, G., Hjorth, J., Jakobsson, P., Christensen, L., \& Holland, S. T. 2001, ApJ, 552, L121

Björnsson, G., Hjorth, J., Pedersen, K., \& Fynbo J. P. U. 2002, ApJ, 579, L59

Cardelli, J. A., Clayton, G. C., \& Mathis, J. S. 1989, ApJ, 345, 245

Castro-Tirado, A. J., Gorosabel, J., Augusteijn, T., \& Castro Cerón, J. M. 2002, $\mathrm{GCN}^{7} \# 1327$

Chevalier, R. A., \& Li, Z. Y. 2000, ApJ, 536, 195

Covino, S., Lazzati, D., Ghisellini, G., et al. 1999, 348, L1

Covino, S., Ghisellini, G., Saracco, P., et al. 2002a, GCN \#1337

Covino, S., Ghisellini, G., Saracco, P., et al. 2002b, GCN \#1345

Covino, S., Lazzati, D., Malesani, D., et al. 2002c, A\&A, 392, 865

Covino, S., Malesani, D., Ghisellini, G., et al. 2002d, GCN \#1498

Covino, S., Ghisellini, G., Malesani, D., et al. 2002e, GCN \#1595

Covino, S., Malesani, D., Ghisellini, G., et al. 2003, A\&A, 400, L9

Dado S., Dar A., \& De Rújula, A. 2002, A\&A, 393, L25

Dai, Z. G., \& Lu, T. 1999, ApJ, 519, L155

Dermer, C. D. 2002, ApJ, submitted [astro-ph/0211300]

Di Serego Alighieri, S. 1997, in Instrumentation for Large Telescopes - VII Canary Islands Winter School of Astrophysics, ed. J. M. Rodriguez de Espinosa, A. Herrero, \& F. Sánchez (Cambridge: Cambridge Univ. Press), 287

Esin, A. A., \& Blandford, R. D. 2000, ApJ, 534, L151

Fruchter, A. S., \& Hook, R. N. 2002, PASP, 114, 144

Fukugita, M., Shimasaku, K., \& Ichikawa, T. 1995, PASP, 107, 945

Galama, T. J., Vreeswijk, P. M., van Paradijs, J., et al. 1998, Nature, 395,670

Gal-Yam, A., Ofek, E. O., \& Lipkin, Y. 2002a, GCN \#1335

Gal-Yam, A., Ofek, E. O., \& Shemmer, O. 2002b, MNRAS, 332, L73

Ghisellini, G., \& Lazzati, D. 1999, MNRAS, 309, L7

Gorosabel, J., Fynbo, J. P. U., Hjorth, J., et al. 2002, A\&A, 384, 11

Hamuy, M., Walker, A. R., Suntzeff, N. B., et al. 1992, PASP, 104, 533

Hamuy, M., Suntzeff, N. B., Heathcote, S. R., et al. 1994, PASP, 106, 566

Harrison, F. A., Yost, S. A., Sari, R., et al. 2001, ApJ, 559, 123

Hiltner, W. A. 1956, ApJS, 2, 389

Hjorth, J., Björnsson, G., Andersen, M. I., et al. 1999, Science, 283, 2073

Hjorth, J., Fynbo, J. P. U., Pian, E., et al. 2002, GCN \#1329

Holland, S. T., Soszynski, I., Gladders, M. D., et al. 2002, AJ, 124, 639

Holland, S.T., Weidinger, M., Fynbo, J. P. U., et al. 2003, AJ, 125, 2291

Horne, K. 1986, PASP, 98, 609

Hurley, K., Cline, T., Frontera, F., et al. 2002, GCN \#1325

Jakobsson, P., Hjorth, J., Fynbo, J. P. U., et al. 2002, A\&A, submitted

Kennicutt, R. C. Jr. 1998, ARA\&A, 36, 189

Krist, J. 1993, in Astronomical Data Analysis Software and Systems II, ed. R. J. Hanisch, R. J. V. Brissenden, \& J. Barnes, ASP Conf. Ser., 52, 536

Kobulnicky, H. A., Kennicutt, R. C. Jr., \& Pizagno, J. L. 1999, ApJ, 514,544

Kumar, P., \& Piran, T. 2000, ApJ, 532, 286

Landolt, A. U. 1992, AJ, 104, 340

Loeb, A., \& Perna, R. 1998, ApJ, 495, 597

Masetti, N., Bartolini, C., Bernabei, S., et al. 2000, A\&A, 359, L23

${ }^{7}$ GCN Circulars are available at:

http://gcn.gsfc.nasa.gov/gcn/gcn3_archive.html 
Masetti, N., Palazzi, E., Pian, E., et al. 2001, A\&A, 374, 382

Masetti, N., Palazzi, E., Pian, E., et al. 2002a, GCN \#1330

Masetti, N., Palazzi, E., Maiorano, E., et al. 2002b, GCN \#1375

Mazzali, P. A., Deng, J., Maeda, K., et al. 2002, ApJ, 572, L61

Medvedev, M. V., \& Loeb, A. 1999, ApJ, 526, 697

Metzger, M. R., Djorgovski, S. G., Kulkarni, S. R., et al. 1997, Nature, 387, 878

Mirabal, N., Paerels, F., \& Halpern, J. P. 2003, ApJ, 587, 128

Møller, P., Fynbo, J. P. U., Hjorth, J., et al. 2002, A\&A, 396, L21

Nakano, S., Kushida, R., \& Li, W. 2002, IAU Circ., 7810

Nakar, E., Piran, T., \& Granot, J. 2003, New Astron., 8, 495

Paczyński, B. 1998, ApJ, 494, L45

Palazzi, E., Masetti, N., Pian, E., et al. 2002, GCN \#1328

Pandey, S. B., Anupama, G. C., Sagar, R., et al. 2003, MNRAS, 340, 375

Patat, F., Cappellaro, E., Danziger, J., et al. 2001, ApJ, 555, 900

Persson, S. E., Murphy, D. C., Krzeminski, W., Roth, M., \& Rieke, M. J. 1998, AJ, 116, 2475

Price, P. A., Schmidt, B. P., \& Axelrod, T. S. 2002a, GCN \#1326

Price, P. A., Schmidt, B. P., \& Axelrod, T. S. 2002b, GCN \#1333

Price, P. A., Kulkarni, S. R., Berger, E. J., et al. 2002c, ApJ, 571, L121

Price, P. A., Kulkarni, S. R., Berger, E. J., et al. 2003, ApJ, in press [astro-ph/0208008]

Ramaprakash, A. N. 1998, Ph.D. Thesis, Inter-University Centre for Astronomy and Astrophysics

(http://www . iucaa.ernet.in/ anr/thesis.html)

Ramirez-Ruiz, E., Dray, L. M., Madau, P., \& Tout, C. A. 2001, MNRAS, 327, 829

Reichart, D. E. 2001, ApJ, 554, 643

Rhoads, J. E. 1999, ApJ, 525, 737

Rol, E., Wijers, R. A. M. J., Vreeswijk, P. M., et al. 2000, ApJ, 544, 707
Rol, E., Wijers, R. A. M. J., Fynbo, J. P. U., et al. 2003, A\&A, submitted

Rybicki, G. B., \& Lightman, A. P. 1979. Radiative Processes in Astrophysics (New York: J. Wiley \& Sons)

Sahu, K. C., Vreeswijk, P. M., Bakos, G., et al. 2000, ApJ, 540, 74

Sari, R. 1999, ApJ, 524, L43

Sari, R., Piran, T., \& Narayan, R. 1998, ApJ, 497, L17

Sari, R., Piran, T., \& Halpern, J. P. 1999, ApJ, 519, L17

Savaglio, S., Fall, S. M., \& Fiore, F. 2003, ApJ, 585, 638

Schlegel, D. J., Finkbeiner, D. P., \& Davis, M. 1998, ApJ, 500, 525

Serkowski, K., Mathewson, D. L., \& Ford, V. L. 1975, ApJ, 196, 261

Simmons, J. F. L., \& Stewart, B. G. 1985, A\&A, 142, 100

Simoncelli, A., Maiorano, E., Palazzi, E., et al. 2002, GCN \#1388

Šimon, V., Hudec, R., Pizzichini, G., \& Masetti, N. 2001, A\&A, 377, 450

Skrutskie, M. F., Schneider, S. E., Stiening, R., et al. 1997, in The Two Micron All Sky Survey (2MASS): Overview and Status, The Impact of Large Scale Near-IR Sky Surveys, ed. F. Garzon, N. Epchtein, A. Omont, B. Burton, \& P. Persi (Dordrecht: Kluwer Acad. Publ.), 25

Stetson, P. B. 1987, PASP, 99, 191

Szeifert, T. 2001, http://www. eso.org/instruments/fors1 /pola.html

Wang, L., Baade, D., Hoeflich, P., \& Wheeler, J. C. 2003, ApJ, submitted [astro-ph/0301266]

Wang, X., \& Loeb, A. 2000, ApJ, 535, 788

Wardle, J. F. C., \& Kronberg, P. P. 1974, ApJ, 194, 249

Waxman, E. 1997, ApJ, 491, L19

Wijers, R. A. M. J., Vreeswijk, P. M., Galama, T. J., et al. 1999, ApJ, 523, L33 